\title{
Structure determination of small vanadium clusters by density-functional theory in comparison with experimental far-infrared spectra
}

\author{
C. Ratsch ${ }^{\text {a) }}$ \\ Fritz-Haber-Institut der Max-Planck-Gesellschaft, Faradayweg 4-6, D-14195 Berlin, Germany \\ and Department of Mathematics and California NanoSystems Institute, University of California, \\ Los Angeles, California 90095-1555
}

A. Fielicke

Fritz-Haber-Institut der Max-Planck-Gesellschaft, Faradayweg 4-6, D-14195 Berlin, Germany and FOM Institute for Plasma Physics "Rijnhuizen," Edisonbaan 14, NL-3439 MN Nieuwegein, The Netherlands

\begin{abstract}
A. Kirilyuk
Institute for Molecules and Materials, Radboud Universiteit Nijmegen, Toernooiveld 1, 6525 ED Nijmegen, The Netherlands
\end{abstract}

J. Behler, G. von Helden, G. Meijer, and M. Scheffler

Fritz-Haber-Institut der Max-Planck-Gesellschaft, Faradayweg 4-6, D-14195 Berlin, Germany

(Received 7 December 2004; accepted 6 January 2005; published online 25 March 2005)

\begin{abstract}
The far-infrared vibrational spectra for charged vanadium clusters with sizes of 3-15 atoms have been measured using infrared multiple photon dissociation of $\mathrm{V}_{n}^{+} \mathrm{Ar} \rightarrow \mathrm{V}_{n}^{+}+\mathrm{Ar}$. Using density-functional theory calculations, we calculated the ground state energy and vibrational spectra for a large number of stable and metastable geometries of such clusters. Comparison of the calculated vibrational spectra with those obtained in the experiment allows us to deduce the cluster size specific atomic structures. In several cases, a unique atomic structure can be identified, while in other cases our calculations suggest the presence of multiple isomers. () 2005 American Institute of Physics. [DOI: 10.1063/1.1862621]
\end{abstract}

\section{INTRODUCTION}

Small clusters or nanoparticles exhibit properties that are often quite different from those in the bulk phase. For example, small metal clusters have been shown to exhibit unusual magnetic properties. ${ }^{1}$ In particular, vanadium clusters have been predicted to have large magnetic moments. ${ }^{2-6}$ Small nanoparticles also play an increasingly important role in catalysis. ${ }^{7,8}$ With new approaches in synthesis, it may become feasible to control the size and possibly also the structure of the nanoparticles, and thus to control their properties. Therefore, it is paramount to gain a better understanding of the atomic structure and properties of small metal clusters.

Direct experimental measurements of the atomic arrangements of small metal clusters are difficult. Various isomers may exist that are stable or metastable configurations. Recently, information on the geometric structure of $\mathrm{Ag}$ and $\mathrm{Au}$ clusters has been obtained by measuring their collision cross sections in an ion mobility experiment. ${ }^{9}$ However, this approach only gives information about the size, but not about the internal structure of the clusters. Optical and photoelectron spectroscopies allow for the determination of the electronic structure, which gives indirect information on the atomic structure. In a few cases, vibrational resolution has been obtained with such techniques, but this results only in a small section of the vibrational spectrum. In principle, the determination of the vibrational spectra is desirable, since it

${ }^{a)}$ Electronic mail: cratsch@math.ucla.edu maps the forces between the atoms and thereby reflects the structure of the cluster. Raman spectroscopy has been applied to some small mass selected clusters, which are accumulated and embedded in rare gas matrices. ${ }^{10,11}$ Nevertheless, the requirement of mass selection limits the practicability of this method and, additionally, the matrix can affect the properties of the embedded clusters.

In order to determine the structure of the clusters, such experiments need to be complemented with theoretical studies. Density-functional theory (DFT) calculations can be used to compare the total energy of a number of cluster isomers of different geometric and electronic structures, thus predicting the energetically preferred structure. For example, Grönbeck and Rosén ${ }^{12}$ performed DFT calculations to predict the ground state structure of neutral vanadium clusters of size 2 to 8 . In a later study, Wu and Ray ${ }^{13}$ presented DFT results for neutral and cationic vanadium clusters in the size range from 2 to 9 . A recent DFT study by Li et al. ${ }^{14}$ reports results for neutral, anionic, and cationic vanadium clusters of sizes up to 8 . Studies that are based on empirical potentials ${ }^{15}$ and the tight binding method ${ }^{16,17}$ have looked at the optimized atomic structure for even larger neutral vanadium clusters.

However, the isomer that is calculated to have the lowest energy is not necessarily the one that is actually present in the experiments. There are several reasons for this: several structures may be very close in energy, so that present-day exchange-correlation functionals are not sufficiently accurate 


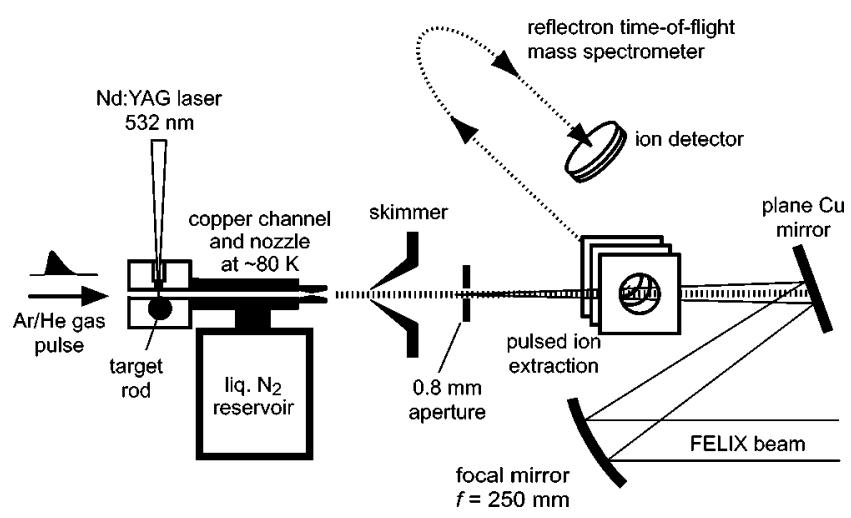

FIG. 1. Scheme of the experimental setup for the IR multiple photon dissociation spectroscopy of the argon complexes of metal cluster cations.

to decide which of them may be the most favorable. Second, for kinetic reasons, the experiment may favor metastable structures.

In this paper we present the details of a combined theoretical and experimental study on the structure determination of metal clusters. We have recently shown that multiple photon dissociation spectroscopy on metal cluster rare gas complexes allows for the determination of cluster size specific far-infrared spectra, and that a comparison with vibrational spectra calculated by DFT can be used to determine the atomic structure, ${ }^{18}$ because these spectra are typically rather different for different atomic configurations. The experimental setup and experimental details are described in Sec. II. The computational procedure and details are given in Sec. III. Finally, in Sec. IV we give a detailed comparison for all the vanadium clusters of size 3 to 15 studied here, and illustrate how the combination of the ground state energy and the comparison of the IR spectrum allows us to identify the atomic structure for most of these clusters.

\section{THE EXPERIMENT}

The experiments are carried out in a molecular beam setup that is coupled to the beam line of the Free Electron Laser for Infrared experiments (FELIX). This laser can produce intense, several microseconds long pulses of tunable IR radiation in the range of $\sim 40-2000 \mathrm{~cm}^{-1}$. Each pulse consist of a train of $\sim 0.3-3$ ps long micropulses of typically $\sim 10 \mu \mathrm{J}$, spaced by $1 \mathrm{~ns}$. The time and intensity profile of the radiation makes FELIX a suitable tool for studying multiple photon excitation processes in molecules or clusters. ${ }^{19}$ Some details of the experimental setup have already been given in Ref. 18, and will also be described more extensively elsewhere. ${ }^{20}$ In short, cationic vanadium clusters $\mathrm{V}_{n}^{+}$are formed in a laser vaporization cluster source by ablating a vanadium target and quenching the plasma with a short pulse of a gas mixture of $0.5 \% \mathrm{Ar}$ in $\mathrm{He}$ (see Fig. 1). Complexes with $\mathrm{Ar}$ atoms $\left(\mathrm{V}_{n}^{+} \mathrm{Ar}\right)$ are formed after passing through a copper channel that is cooled to about $80 \mathrm{~K}$. The molecular beam containing these complexes is overlapped with the far-IR output of FELIX. Resonant absorption of one or multiple IR photons by the complexes can be followed by evaporation of single or more Ar atoms from the complex leading to decreases in their abundances. These complex abundances are measured using a reflectron time-of-flight mass spectrometer as a function of the IR wavelength. After normalization for laser power variations and intensity fluctuations stemming from the cluster source, one obtains the absorption spectra of the corresponding vanadium cluster complexes. Although the thus obtained spectra correspond to the spectra of the Ar complexes, the vanadium clusters are the active chromophore and the influence of the Ar atoms is assumed and expected to be negligible. The spectra are recorded in the frequency range where one expects the metal cluster vibrations, between 140 and $450 \mathrm{~cm}^{-1}$.

The IR induced changes in the complex intensities are converted to absorption cross sections $\sigma(\nu)$ and normalized for variations of the laser power $P(\nu)$ over the tuning range using

$$
\sigma(\nu)=\ln \left[I_{0} / I(\nu)\right] / P(\nu),
$$

where $I(\nu)$ and $I_{0}$ are the intensities of a certain $\mathrm{V}_{n} \mathrm{Ar}^{+}$complex with and without FELIX irradiation, respectively. This procedure assumes a one-photon absorption process. However, the binding energies of Ar atoms are on the order of 0.1 $\mathrm{eV}^{21,22}$ which implies that the dissociation follows the absorption of multiple photons. In addition, the spectral width and shape of the focussed beam are changing with wavelength, and it is therefore not at all clear if this simple normalization suffices.

Most of the experimental spectra are obtained on the monoargon complex, and especially for the larger clusters the spectra do not depend on the number of attached argon atoms. However, for some complexes we observe distortion of spectral features or disappearance of some bands because of formation of those complexes as a result of fragmentation of higher argon complexes. This effect appears mainly at lower frequencies where the IR laser intensity is relatively low and the excitation does not necessarily lead to complete evaporation of all argon atoms. Therefore, for some clusters $(n=5-7)$, spectra of complexes with more argon atoms are used as these complexes suffer less from the interference of dissociation of larger clusters.

In addition to experimental effects, the appearance of the IR spectra can be influenced by the mechanism of multiple photon absorption. The successive resonant absorption of multiple photons depends on a fast intramolecular vibrational energy redistribution. The time scale for this process is much faster than the duration of the excitation pulse $(\sim 5 \mu \mathrm{s})$ and therefore the absorption/redistribution cycle can be accomplished up to several hundred times for one cluster during a single pulse. However, vibrational energy redistribution depends on a high density of vibrational states and for the smallest clusters it can be inefficient, especially at low energy. This can affect the intensities of low energy bands or even lead to their disappearance. Furthermore, the (cross) anharmonicities can lead to small redshifts of the absorption bands relative to the bands in single photon absorption spectra. $^{23}$ 


\section{DENSITY-FUNCTIONAL THEORY CALCULATIONS}

\section{A. Computational procedure}

The DFT calculations are carried out using the $\mathrm{DMOL}^{3}$ code, ${ }^{24}$ which is an all-electron code that uses numerical atomic orbitals as a basis set. It is an extremely efficient code for small clusters. In all calculations shown below, we use an all-electron basis set that consists of 24 basis functions for each atom. The basis functions are truncated at a real space cutoff radius of 12 bohrs. Details of the convergence tests are given in the Appendix.

We employed the following computational procedure: First, we calculated the ground state energy for a large number of geometries for the clusters of each size. The atomic positions of the structures were always relaxed. As initial geometries we started with all the structures that have been discussed previously in the literature. ${ }^{12-17}$ In addition, we tried a large number of different geometries that were derived from previously calculated structures. For example, for many clusters one can describe the geometry as a superposition of smaller building blocks that typically are trigonal, tetragonal, pentagonal, or hexagonal pyramids. Thus, one can often simply add or subtract an atom from a cluster of size $n$ to obtain a start geometry for a cluster of size $n+1$ or $n-1$. In this manner, we typically generated at least ten or more different geometries for each size. It is important to emphasize that finding the correct initial geometry is a major challenge. As the cluster size increases, the number of possible atomic configurations increases dramatically. Therefore, it is possible that for certain cluster sizes (in particular, the larger ones) we did not find the correct atomic structure. We also note that it is important to not enforce any symmetry. In fact, slightly distorted structures are often more favorable than highly symmetric ones.

We also tested the different possible spin states for all clusters. The effect of the spin state will be illustrated below. Once the structures and spin states that are energetically most favorable were detected, we calculated the vibrational spectrum of these systems. This was done by displacing each atom in each direction, in order to evaluate the $3 n$ dimensional force-constant matrix. Then we diagonalized the resulting dynamical matrix. The IR intensities were obtained from the derivative of the dipole moment. For the purpose of an easy comparison to the experimental data, we folded for all results shown in Sec. IV the calculated spectra with a Gaussian line shape function of half-width of $2 \mathrm{~cm}^{-1}$.

\section{B. The exchange-correlation functional}

All results presented in Sec. IV were obtained using the generalized gradient approximation (GGA) in the parametrization of Perdew, Burke, and Ernzerhof (PBE) (Ref. 25) for the exchange-correlation (XC) functional. However, an important question that one needs to ask is whether the results for the energy differences between different structures and the calculated vibrational spectra are sensitive to the choice of the XC functional. We have therefore tested the importance of the choice of the XC functional. In Figs. 2 and 3 we compare results obtained with the GGA in the PBE parametrization to results obtained with the GGA in the RPBE

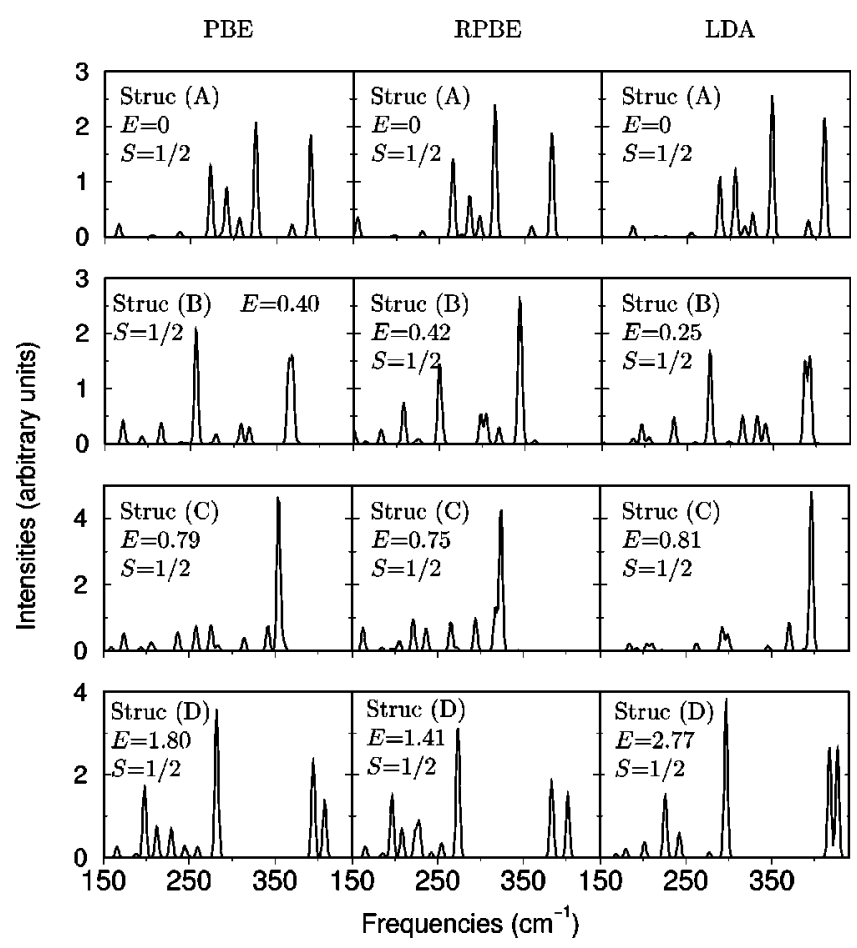

FIG. 2. Comparison of IR spectra for the four most relevant structures for $\mathrm{V}_{8}^{+}$obtained with the GGA within the PBE (left) and the RPBE (middle) parametrization, as well as with the LDA (right). The relative energies are given in $\mathrm{eV}$. Note that no scaling factor has been applied.

parametrization, ${ }^{26}$ and to results in the local-density approximation (LDA). ${ }^{27}$ The geometries were all relaxed for each of the $\mathrm{XC}$ functionals. An agreement of all quantities of interest between these three different $\mathrm{XC}$ functionals is a good indication for the reliability of the theoretical results.

PBE

\begin{tabular}{|l|l|l|}
\hline Struc (A) & Struc (A) & $\begin{array}{l}\text { Struc (A) } \\
E=0 \\
S=0\end{array}$ \\
$S=1$ & $\mid$ & \\
$S=1$
\end{tabular}
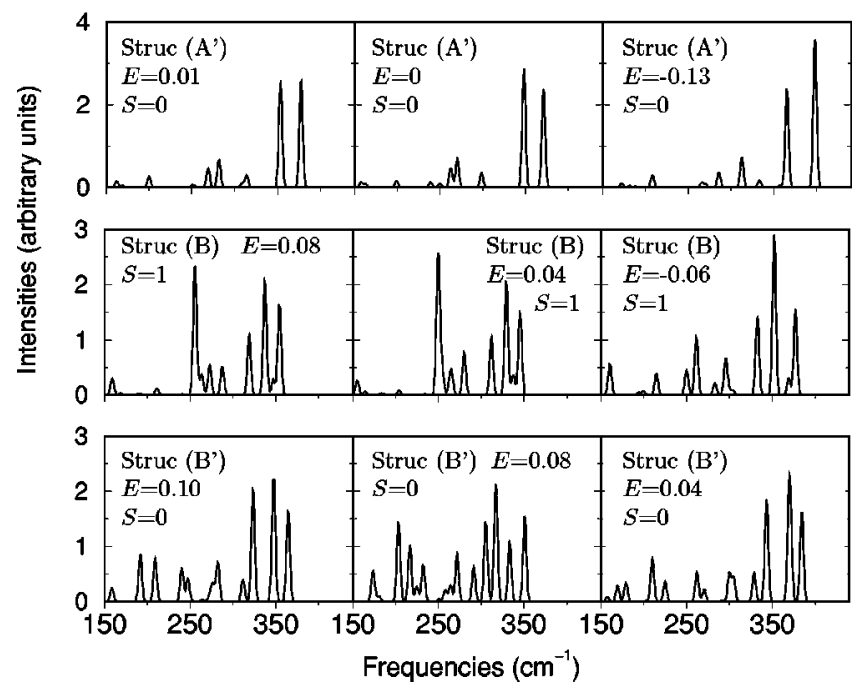

FIG. 3. Comparison of IR pectra for the four most relevant structures for $\mathrm{V}_{9}^{+}$ obtained with the GGA within the PBE (left) and the RPBE (middle) parametrization, as well as with the LDA (right). The relative energies are given in $\mathrm{eV}$. Note that no scaling factor has been applied. 
In Fig. 2 we show the calculated spectra for the four lowest energy structures for $\mathrm{V}_{8}^{+}$, which are labeled $(A)-(D)$. In Fig. 3 we show the calculated spectra for the two lowest energy structures for $\mathrm{V}_{9}^{+}$. They are labeled $(A)$ and $(B)$, and have a spin of $S=1$. The corresponding structures with spin state $S=0$ are labeled $\left(A^{\prime}\right)$ and $\left(B^{\prime}\right)$. The exact atomic configurations are shown in Figs. 10 and 12. We chose these two cluster sizes because they are approximately in the middle of the size range studied in this paper. $\mathrm{V}_{8}^{+}$is very representative for a cluster where the energetically most preferred isomers have rather different atomic structures, while $\mathrm{V}_{9}^{+}$is representative for a cluster where the structural and energetical differences for the lowest energy isomers are rather subtle.

The main observations when comparing the IR spectra obtained with different XC functionals are that for all structures shown, the spectra are very similar. The peaks for the two different GGA parametrizations are almost at the same positions, while the LDA peaks are shifted to larger frequencies. This can easily be explained by the well-known overbinding for the LDA, which leads to larger force constants. But there are subtle differences in the spectra, in particular, in the relative strength of the peaks that are an indication for the accuracy we can expect from this type of calculation. For example, for structure $\left(A^{\prime}\right)$ for $\mathrm{V}_{9}^{+}$the relative intensity of the two main peaks changes by almost $50 \%$ between the different XC functionals. Similar effects can be seen for the other structures as well.

The results for the different $\mathrm{XC}$ functionals also indicate that one has to be particularly careful in the interpretation of the data when two peaks are very close. For example, for structure $(B)$ for $\mathrm{V}_{8}^{+}$, we see a strong peak at $\approx 250 \mathrm{~cm}^{-1}$ and a broader peak at $\approx 360 \mathrm{~cm}^{-1}$ for the PBE-GGA parametrization. This latter peak is actually a double peak, as can be seen best from the LDA results. Similarly, it appears that the spectrum for structure $\left(B^{\prime}\right)$ for $\mathrm{V}_{9}^{+}$is slightly different for different XC functionals. The spacing and relative strengths of the first eight peaks change slightly between the different $\mathrm{XC}$ functionals. Most prominently, it appears that there is one additional peak in the RPBE parametrization. However, close inspection of our results shows that the peak at second largest wave number obtained with the PBE-GGA XC functional (located at $\approx 350 \mathrm{~cm}^{-1}$ ), and the peak at second largest wave number obtained with the LDA functional (located at $\approx 380 \mathrm{~cm}^{-1}$ ) do actually consist of two peaks. We note that peaks that are that close are not possible to distinguish experimentally. These observations will guide us in the interpretation of the experimental spectra (below).

We now turn our attention to the reliability of the calculated energy differences. For structures $(A)-(C)$ for $\mathrm{V}_{8}^{+}$, the energy differences are essentially the same within the PBE and RPBE treatments of the GGA. They are also very similar to the LDA results. The only noticeable difference is structure $(B)$, which is $\approx 0.15 \mathrm{eV}$ more favorable within the LDA. For structure $(D)$ the energy differences to structure $(A)$ are the largest among the different XC functionals. Structure $(D)$ has a much higher relative energy, and different local minima exist, that correspond to different distortions. Using the LDA functional, the system converges to a differently distorted structure upon relaxation of the geometry.
TABLE I. Additional energy contribution (in eV) to the energy differences [relative to structure (A)] due to the vibrational energy as calculated by equation (1) for $\mathrm{V}_{8}^{+}$at different temperatures.

\begin{tabular}{lllll}
\hline \hline Temperature (K) & \multicolumn{1}{c}{0} & 100 & 200 & \multicolumn{1}{c}{300} \\
\hline Struc (A) & 0 & 0 & 0 & 0 \\
Struc (B) & 0.007 & 0.014 & 0.027 & 0.040 \\
Struc (C) & 0.020 & 0.028 & 0.046 & 0.064 \\
Struc (D) & 0.042 & 0.053 & 0.083 & 0.117 \\
\hline \hline
\end{tabular}

For the second system discussed here, $\mathrm{V}_{9}^{+}$, the situation is slightly more complex. The PBE and RPBE parametrization give the same energy ordering for the different structures and the different spins. The differences are very small, less than $0.1 \mathrm{eV}$. However, if we compare the GGA results with the LDA results, we see differences: With the LDA, structure $\left(A^{\prime}\right)$ with spin $S=0$ is favored, and structure $(A)$ with $S=1$ is higher in energy by $0.13 \mathrm{eV}$. The energy differences between $(A)$ and $(B)$ and $\left(A^{\prime}\right)$ and $\left(B^{\prime}\right)$ are similar to the GGA results. We conclude that the energy difference of the same system with different spins is not always well produced when the difference is small, and in fact for this particular case the order is reversed. From these calculations we estimate that the energy difference between different spins has an accuracy of only $0.2 \mathrm{eV}$, while the energy difference between systems with the same spin is accurate to within $0.1 \mathrm{eV}$.

\section{Vibrational-free energies}

The DFT calculations as discussed so far only give the total energy for each system. In this paper, we also include the contributions of the vibrational degrees of freedom to the free energies. The vibrational contribution to the free energies are calculated according to

$$
\begin{aligned}
F^{\mathrm{vib}}(T)= & \sum_{\nu}\left[h \nu\left(\frac{1}{2}+\frac{1}{\exp \left(h \nu / k_{B} T\right)-1}\right)\right] \\
& -\sum_{\nu}\left(\frac{h \nu}{\exp \left(h \nu / k_{B} T\right)-1}\right. \\
& \left.-k T \ln \left[1-\exp \left(-h \nu / k_{B} T\right)\right]\right),
\end{aligned}
$$

where the sums go over all vibrational modes with frequency $\nu, k_{B}$ is the Boltzmann constant, $h$ is Planck's constant, and $T$ is the temperature. The first term corresponds to the vibrational energy, while the second term represents the vibrational entropy. We find that the total contribution to the free energy due to the vibrational terms is typically of the order of several tenths of an $\mathrm{eV}$, but the contribution to the energy differences is much smaller. As an example, the temperature dependence of the vibrational energy for $\mathrm{V}_{8}^{+}$is shown in Table I for different structures. We get similar (or even smaller) contributions for all other clusters. In fact, with the exception of structure $(D)$ for $\mathrm{V}_{8}^{+}$, this additional contribution is less than $0.1 \mathrm{eV}$ for all systems discussed in this paper, for temperatures up to $300 \mathrm{~K}$. In particular, the contribution to the energy differences due the vibrational terms in the free energy is smaller than the accuracy of the calculations, and is 
therefore not relevant. Nevertheless, all energies quoted in this paper include the contributions of the zero-point vibrational energy.

\section{Scaling of the frequencies}

It is a well known problem that the vibrational frequencies that are calculated from DFT are slightly shifted compared to the experimental ones. This is partly related to the fact that the bulk lattice constant is slightly overestimated (underestimated) within the GGA (LDA). It is therefore common to apply a scaling factor to the calculated frequencies to bring the calculated spectra in better agreement with the ones measured experimentally. We apply a common, constant factor of 0.87 to all the results shown in Sec. IV. This factor is obtained from the calculations for the vanadium dimer: We calculate a frequency of $618 \mathrm{~cm}^{-1}$ for $\mathrm{V}_{2}$ using the GGA with $\mathrm{PBE}$, which is in good agreement with the values of $628 \mathrm{~cm}^{-1}$ and $596 \mathrm{~cm}^{-1}$ that were calculated by Grönbeck and Rosén ${ }^{12}$ and Calaminici et al., ${ }^{28}$ where the LDA with gradient corrections, and the GGA were used, respectively. The experimental value ${ }^{29}$ is $538 \mathrm{~cm}^{-1}$, so the ratio of the experimental value to the one calculated by us is 0.87 . We note that a scaling factor of 0.89 would result for GGA with RPBE, and a smaller scaling facor of 0.82 would result for the LDA. A smaller scaling factor for the LDA is consistent with the well known overbinding. The bond length for $\mathrm{V}_{2}$ was calculated to be $1.77 \AA$, in excellent agreement with the experimental value ${ }^{30}$ of $1.77 \AA$, and with previous DFT results of $1.77 \AA$ and $1.802 \AA$ by Grönbeck and Rosén ${ }^{12}$ and Calaminici et al. ${ }^{28}$ respectively. We also note that the calculated bond length for $\mathrm{V}_{2}^{+}$of $1.76 \AA$ agrees very well with the experimental value ${ }^{31}$ of $1.735 \AA$.

We apply the same scaling factor of 0.87 to all frequencies shown, even though it is conceivable that this scaling factor should be slightly different for clusters of different size, or different symmetry. When comparing the experimental to the theoretical spectra it is therefore more relevant to focus on the (relative) spacing between different peaks in the spectrum, rather than on the agreement of the absolute frequencies.

\section{E. The Effect of the rare gas atoms}

All the calculations presented below are for $\mathrm{V}_{n}^{+}$clusters, while the experimental spectra are obtained from the dissociation of $\mathrm{V}_{n} \mathrm{Ar}^{+} \rightarrow \mathrm{V}_{n}^{+}+$Ar. This is justified, because the rare gas atoms bind only very weakly to the $\mathrm{V}_{n}^{+}$cluster. Nevertheless, we did calculations to check this assumption. For $\mathrm{V}_{3}^{+}$ and $\mathrm{V}_{4}^{+}$, we have calculated the vibrational spectra of the lowest energy structures with an additional Ar atom attached. We find that the calculated frequencies as well as the relative line intensities are essentially unaffected (changes are less than $3 \mathrm{~cm}^{-1}$ ), and that the binding energy of the Ar atom is only about $0.1 \mathrm{eV}$.

\section{RESULTS: COMPARISON OF CALCULATIONS WITH EXPERIMENT}

In Sec. IV A we will discuss some general trends. Then, we will present results for all clusters containing from 3 to

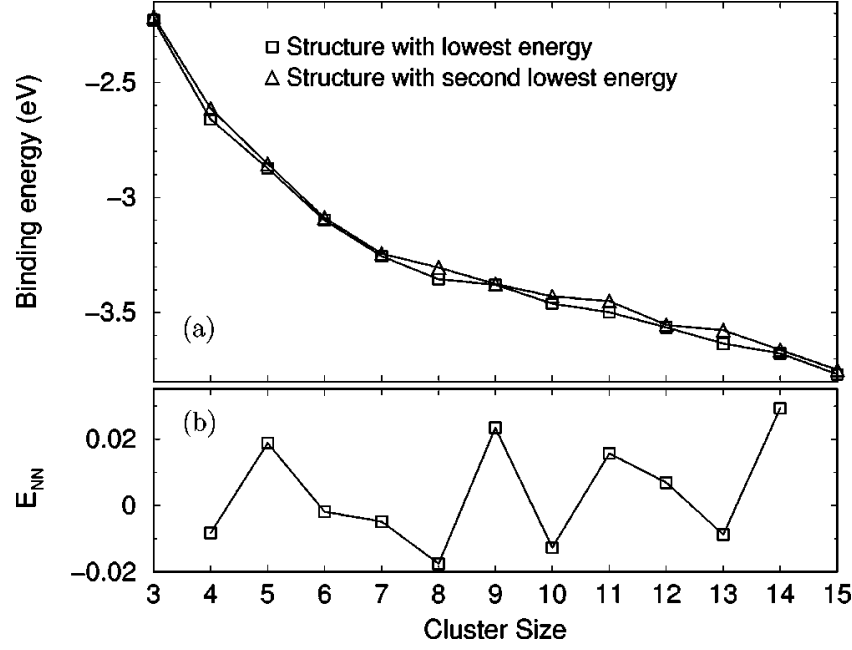

FIG. 4. (a) Binding energy per atom for the two most stable geometries for $\mathrm{V}_{5}^{+}-\mathrm{V}_{15}^{+}$. (b) Discrete second derivative of the binding energy per atom $E_{\mathrm{NN}}$ for the most stable structures.

15 atoms in Sec. IV B-IV N. For each of these subsections we will show one key figure that includes the structure representation of the most stable configurations at the top, the experimentally measured spectrum below, and the calculated spectra for all the relevant structures underneath. We will always consider at least two different geometric structures and often several different spin states. In the discussion below, structures that have almost the same geometry, but a different spin state, will also be referred to as different structures. The spin state of the structures considered, as well as the energy difference (in $\mathrm{eV}$ ) relative to the most stable structure are noted in the figure.

\section{A. General trends}

Our theoretical results clearly show that each cluster has a very distinct vibrational spectrum. Some clusters exhibit very few peaks, while other clusters (that might differ in the number of atoms by just one) have many peaks, indicating a significant change in the symmetry of the atomic arrangement.

In Fig. 4(a) we show the binding energy per atom as a function of the cluster size. It decreases monotonically, and approaches the value of the cohesive energy. The latter has been calculated for neutral vanadium bulk in the bcc structure as $E_{\text {coh }}=-4.60 \mathrm{eV}$, which is larger than the experimental value of $-5.31 \mathrm{eV}$. The energy decrease is rather smooth; in particular, the binding energy per atom does not exhibit strong quantum size effects or large jumps in the energy. Only upon close inspection one might argue that the decrease in energy per atom is particularly strong for the clusters of size 8,10 , and 13 , indicating that these are more stable (relatively) than the other sizes. This can also be seen in Fig. 4(b), where we plot the discrete second derivative of the binding energy for each cluster $E_{N N}(i)=[E(i-1)-2 E(i)+E(i+1)] / 2$, which has minima for sizes 8,10 , and 13 .

Our results show that the structures with the lowest energy are also the ones with the lowest possible spins. Since vanadium has 23 electrons, and we consider cationic clusters in this study, this means that we typically get spin $S=0$ for 
(a)

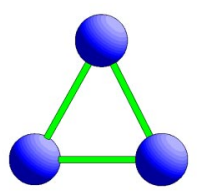

$(\mathrm{A}, \mathrm{B}, \mathrm{C})$

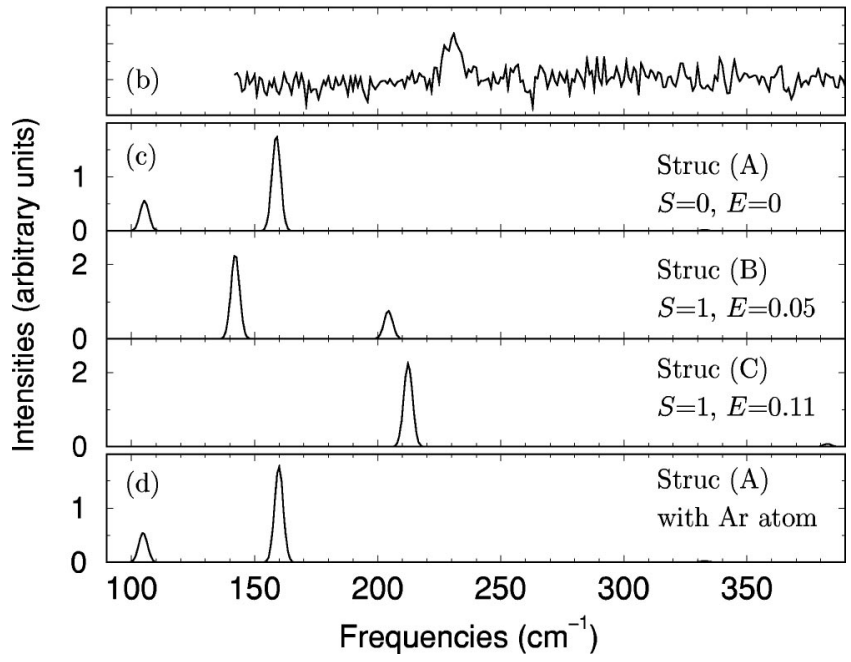

FIG. 5. Results for $\mathrm{V}_{3}^{+}$. (a) Schematic representation of the three most stable structures $(A)-(C)$. Since they are very similar (and only differ slightly in bond lengths), only one picture is shown; (b) the spectrum measured experimentally; (c) the spectrum calculated for the structures $(A)-(C)$; (d) the spectrum of structure $(A)$ with an $\mathrm{Ar}$ atom attached. The energy differences shown are in $\mathrm{eV}$.

clusters with an odd number of atoms, and $S=1 / 2$ for clusters with an even number of atoms. This is in agreement with experimental results for neutral vanadium clusters, where it has been found that at low temperatures the low spin structures are preferred. ${ }^{32}$ The only exception in our calculations is $\mathrm{V}_{5}^{+}$. There, however, the energy differences of structures $(A)$ and $(B)$ with $S=1$ to the same structures with $S=0$ are found to be within the accuracy of our calculations, so that we cannot predict with certainty which of these four systems will be the true ground state structure.

\section{B. Results and discussion for $V_{3}^{+}$}

We find several local minima for $\mathrm{V}_{3}^{+}$. Our calculations indicate that an isosceles triangle with bond lengths $2.14 \AA$, $2.14 \AA$, and $2.28 \AA$, with a spin of $S=0$ is preferred [structure (A) in Fig. 5(a)]. A similar triangle with bond lengths $2.15 \AA$, $2.15 \AA$, and $2.24 \AA$ with $S=1$ is slightly higher in energy [structure $(B)]$. We also discuss a third structure $(C)$, where the isosceles are longer than the base $(2.24 \AA$, $2.24 \AA$, and $2.04 \AA$, respectively), with a spin $S=1$. The calculated spectra for all three structures are shown in Fig. 5(c). In the calculations we find that the spectra are dominated by one strong peak, that is shifted slightly, depending on the aspect ratio of the triangular bonds. It is expected that for the trimer in $C_{2 \nu}$ symmetry, all three vibrational modes are infrared active, whereas in $D_{3 h}$ symmetry only one mode is infrared active.

Within the experimentally studied frequency range only one peak at $231 \mathrm{~cm}^{-1}$ has been found. This would be close to the calculated band positions of $204 \mathrm{~cm}^{-1}$ for structure $(B)$ or
$210 \mathrm{~cm}^{-1}$ for structure $(C)$. A second vibrational transition is known from earlier pulsed field ionization zero kinetic energy (ZEKE) photoelectron spectroscopy experiments by Yang and co-workers. ${ }^{33,34}$ In their spectra a feature $172 \mathrm{~cm}^{-1}$ apart from the band origin has been attributed to a transition between the ground electronic state of $\mathrm{V}_{3}$ to the first excited level of a totally symmetric vibration mode of the ground electronic state of $\mathrm{V}_{3}^{+}$. However, this interpretation may not be appropriate if the ground state structures of neutral and cationic trimer are very different. We do not observe a peak at this wave number and it is not clear if this is due to a low IR intensity (or even IR inactivity in case of $D_{3 h}$ symmetry) of this mode or if its absence is related to the low density of vibrational states of $\mathrm{V}_{3}^{+}$at low energy (see above).

If only the experimental vibrational frequencies are compared to the calculated values for the different structures, the presence of $(A)$ is rather unlikely since it has no transition around $230 \mathrm{~cm}^{-1}$. Structure $(B)$ fits much better with transitions at $142 \mathrm{~cm}^{-1}$ and at $204 \mathrm{~cm}^{-1}$; however, these frequencies are both about $30 \mathrm{~cm}^{-1}$ too low when compared to the ZEKE and IR experiments, respectively. Also $(C)$ would be in rather good agreement with a frequency at $212 \mathrm{~cm}^{-1}$. Additional modes are found for $(A),(B)$, and $(C)$ at $333 \mathrm{~cm}^{-1}$, $333 \mathrm{~cm}^{-1}$, and $382 \mathrm{~cm}^{-1}$, respectively, but these have only low IR intensity.

Due to the small energy differences of the three calculated structures, and the uncertainty in the calculated band positions, we cannot unambiguously identify which bond lengths and aspect ratio of the triangular structure is seen in the experiment.

In previous studies ${ }^{12,13}$ an isosceles triangle for the neutral vanadium trimer has been suggested. An isosceles triangle for neutral and charged trimers has been reported by $\mathrm{Li}$ et al. ${ }^{14}$ However, our results do not agree with the calculations of $\mathrm{Wu}$ and $\mathrm{Ray}^{13}$ and Calaminici et al., ${ }^{28}$ who found that an equilateral triangle is preferred for the cationic trimer. We note that Calaminici et al. also discuss an isosceles triangle (that is slightly higher in energy) that has almost identical bond length as our structure $(A)$. We have carefully investigated this issue, and found that any equilateral structure relaxes into one of the isosceles structures. We note that the linear trimer structure (not shown here) is significantly higher in energy (and in fact is not stable upon small distortions).

We have also tested the effect of the Ar atom on the calculated vibrational spectrum for $\mathrm{V}_{3}^{+}$for structure $(A)$. We find that the Ar atom is bound very weakly with $\approx 0.1 \mathrm{eV}$. The position of the Ar atom is in the plane of the triangle above the apex. The vibrational spectrum is almost identical, as can be seen in Fig. 5(d). In particular, the main peak is still at $159 \mathrm{~cm}^{-1}$, just as for structure $(A)$ without an Ar atom. In the spectrum of the $\mathrm{V}_{3} \mathrm{Ar}^{+}$complex there are two additional peaks at low frequencies, corresponding to the weakly bound Ar atom. We therefore confirm that the Ar atom has essentially no effect on the vibrations of the $\mathrm{V}_{3}^{+}$ion.

\section{Results and discussion for $\mathrm{V}_{4}^{+}$}

We find that a trigonal pyramid as shown in Fig. 6(a) is the energetically preferred structure for $\mathrm{V}_{4}^{+}$. The result is in 
(a)

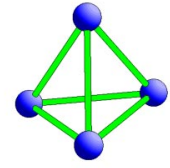

$(\mathrm{A}, \mathrm{B}, \mathrm{C})$

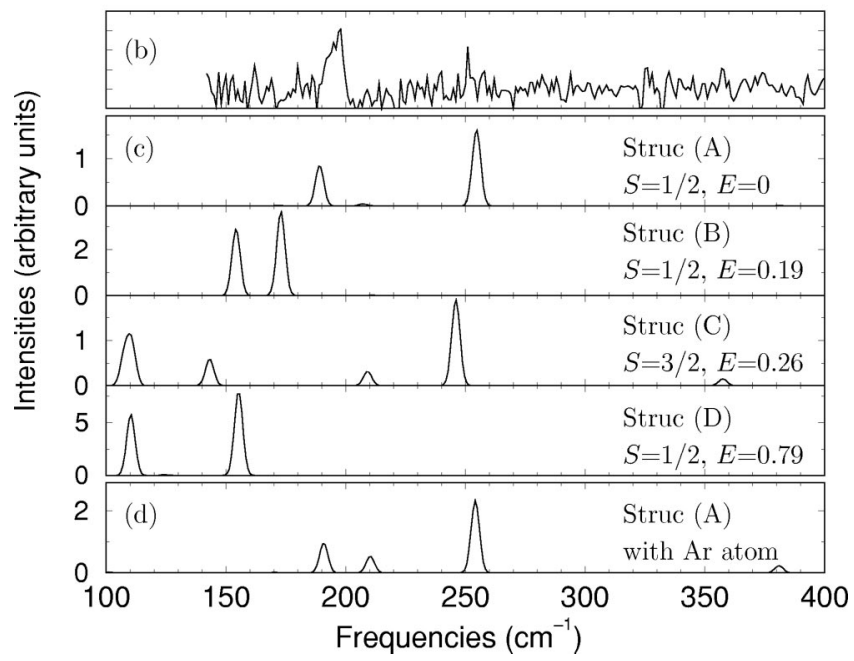

FIG. 6. Results for $\mathrm{V}_{4}^{+}$. (a) Schematic representation of the four most stable structures $(A)-(D)$. Since structures $(A)-(C)$ are very similar (and only differ slightly in bond lengths), only one picture is shown; (b) the spectrum measured experimentally; (c) the spectrum calculated for the structures $(A)-(D)$; (d) the spectrum of structure $(A)$ with an $\mathrm{Ar}$ atom attached. The energy differences shown are in $\mathrm{eV}$.

agreement with the calculations of $\mathrm{Wu}$ and Ray. ${ }^{13}$ A planar structure has been predicted for neutral vanadium tetramers ${ }^{12-14}$ and in a recent study for $\mathrm{V}_{4}^{+}$by Li et al. ${ }^{14} \mathrm{We}$ find that a planar structure is much higher in energy for the cations. In Fig. 6(c) we show the calculated spectra of three trigonal pyramids that differ slightly in how they are distorted and thus differ in bond lengths. In addition, we show the calculated spectrum of the planar structure $(D)$. Among the pyramid structures, structure $(A)$ is the most symmetric one, where two (opposite) bonds have lengths of $2.21 \AA$, and $2.29 \AA$, respectively, while the remaining four bonds are 2.32 $\AA$. Structures $(B)$ and $(C)$ are more distorted, with bond lengths of $2.45 \AA, 2.69 \AA$, and $2.25 \AA$ (four times), and 2.13 $\AA$, $2.25 \AA, 2.54 \AA$ (twice), and $2.30 \AA$ (twice), respectively. Structures $(A)$ and $(B)$ have a spin of $S=1 / 2$, while structure (C) has a spin of $S=3 / 2$.

The experimental spectrum [cf. Fig. 6(b)] exhibits a strong peak at $198 \mathrm{~cm}^{-1}$ and another weak peak around $255 \mathrm{~cm}^{-1}$. The later peak appears very noisy, but it is real and can be found also in other data sets. It is found that the positions of the peaks in the spectrum calculated for the most symmetric pyramid [structure $(A)]$ agree very well with the ones observed experimentally. As mentioned above, the agreement of the (relative) positions of the peaks is most significant, and the relative values of the intensities might be less reliable (in the calculations as well as in the experiment). We note that the calculated frequencies for the planar structure $(D)$ (that is less favorable by $0.79 \mathrm{eV}$ ) are significantly lower than the ones observed in the experiment. (a)

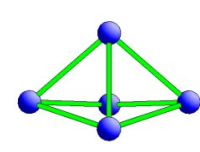

(A)

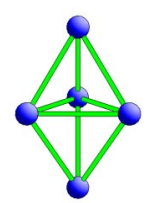

(B)

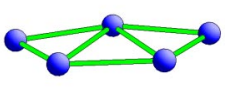

(C)

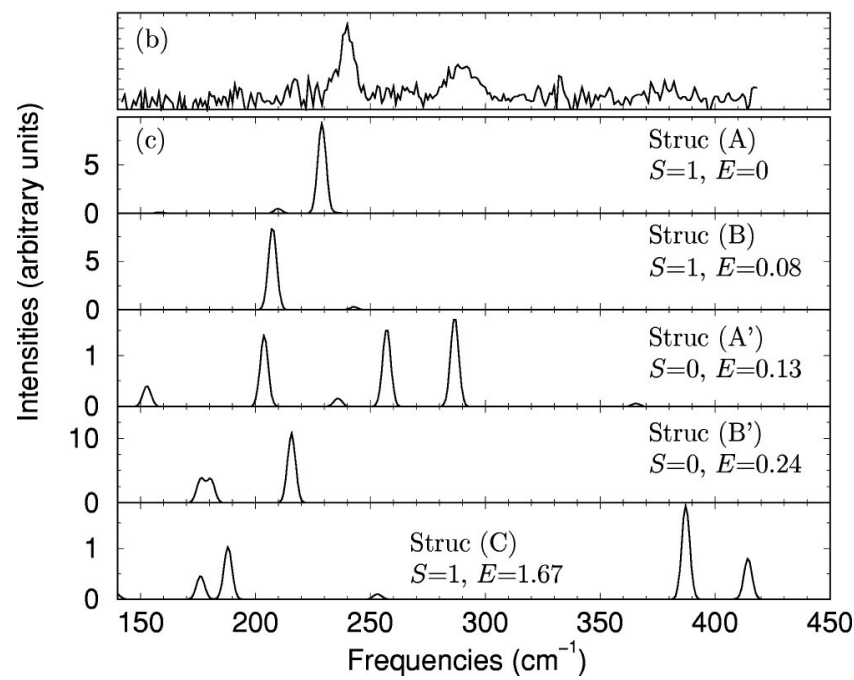

FIG. 7. Results for $\mathrm{V}_{5}^{+}$. (a) Schematic representation of the three most stable structures $(A)-(C)$; (b) the experimental spectrum is constructed from the data for $\mathrm{V}_{5}^{+} \mathrm{Ar}_{2}$ and $\mathrm{V}_{5}^{+} \mathrm{Ar}_{4}$; (c) the spectrum calculated for the structures $(A)$, $(B),\left(A^{\prime}\right),\left(B^{\prime}\right)$, and $(C)$. The energy differences shown are in $\mathrm{eV}$.

We also tested the influence of the Ar atom on the IR spectrum of $\mathrm{V}_{4}^{+}$for structure $(A)$, which is shown in Fig. 6(d). Similarly to the results for $\mathrm{V}_{3}^{+}$, we find that the $\mathrm{Ar}$ is only weakly bound with $\approx 0.1 \mathrm{eV}$, and that the vibrational spectra are very similar for $\mathrm{V}_{4}^{+}$and $\mathrm{V}_{4}^{+} \mathrm{Ar}$. In particular, we find that the two main peaks for structure $(A)$ at $254 \mathrm{~cm}^{-1}$ and $187 \mathrm{~cm}^{-1}$ shift only slightly to $251 \mathrm{~cm}^{-1}$ and $184 \mathrm{~cm}^{-1}$ for $\mathrm{V}_{4}^{+} \mathrm{Ar}$. The only noticeable effect is that the peak at $210 \mathrm{~cm}^{-1}$ is more pronounced.

\section{Results and discussion for $V_{5}^{+}$}

The calculations for $\mathrm{V}_{5}^{+}$reveal that the structure with the lowest energy is a (slightly buckled) tetragonal pyramid with a spin $S=1$ [structure $(A)$ in Fig. 7(a)]. It is $\approx 0.1 \mathrm{eV}$ lower in energy than a trigonal bipyramid with $S=1$ [structure $(B)]$. A spin state that is not the lowest possible spin state has also been reported in optical absorption spectroscopy experiments ${ }^{35}$ for $\mathrm{V}_{5}^{+}$. Both structures exhibit only one peak in the vibrational spectrum, which is shifted slightly to the blue for structure $(B)$. The similarity of the spectra for the two structures is due to the fact that a buckled tetragonal pyramid can be the same as a distorted trigonal bipyramid. Our results are in agreement with previous studies. For cationic, ${ }^{13,14}$ anionic, ${ }^{14}$ and neutral ${ }^{12,14,15} \mathrm{~V}_{5}$, the trigonal bipyramid was found to be energetically preferred. However, the DFT study of Wu and Ray ${ }^{13}$ and a later molecular dynamics study ${ }^{17}$ find the (buckled) tetragonal pyramid to be the energetically preferred structure. 
The same structures $\left(A^{\prime}\right)$ and $\left(B^{\prime}\right)$ with spin $S=0$ are both slightly higher and energy, but have rather different spectra. However, the energy differences in both cases are only about $0.1 \mathrm{eV}$, which is close to the expected accuracy of our calculations, in particular, when different spin states are considered. We also show results for the planar structure $(C)$, which is much higher in energy, and also has a completely different spectrum.

The experimental spectrum for $\mathrm{V}_{5}^{+}$shows a peak at $232 \mathrm{~cm}^{-1}$, and a broad feature around $290 \mathrm{~cm}^{-1}$. The peak at $232 \mathrm{~cm}^{-1}$ is very well reproduced by the calculations for structure $(A)$ [or also structure $(B)]$ with $S=1$. The additional (broad) peak in the experimental data is not found in our calculations for the lowest energy structures $(A)$ and $(B)$ with $S=1$. However, structure $\left(A^{\prime}\right)$ with $S=0$ has two additional peaks, between $250 \mathrm{~cm}^{-1}$ and $290 \mathrm{~cm}^{-1}$, that could match the broad experimental peak around $290 \mathrm{~cm}^{-1}$. Thus, in the IR spectra best agreement is obtained between the experimental spectrum and that of $\left(A^{\prime}\right)$.

\section{E. Results and discussion for $\mathrm{V}_{6}^{+}$}

The most stable structure for $\mathrm{V}_{6}^{+}$is a tetragonal bipyramid. This is in agreement with previous results ${ }^{12,13,15,17}$ that all predict this structure. We have also tested a number of additional structures, but they are all significantly higher in energy. In particular, structure $(D)$ has been suggested previously ${ }^{12,14}$ but our calculations predict this structure to be $0.54 \mathrm{eV}$ higher in energy. However, we find that different local minima for the tetragonal bipyramid exist, that all correspond to slightly different degrees of stretching and distortion from the most symmetric arrangement. All have a spin of $S=1 / 2$ (and the same structures with different spin are all higher in energy).

In Fig. 8 we show the spectra of the four most favorable structures. All tetragonal bipyramids have three major peaks, with one at $245 \mathrm{~cm}^{-1}$, and two more peaks at higher frequencies. The spacings between the peaks depends on the distortions of the structures, with the least distorted structure $(C)$ exhibiting the smallest splitting. Here, the four base bonds (almost horizontal in Fig. 8) are all $2.28 \AA$, while the eight pyramid bonds are alternating between $2.61 \AA$ and $2.31 \AA$. Structure $(B)$ is similar, but with a larger aspect ratio: the four base bonds are $2.26 \AA$, while the pyramid bonds are $2.63 \AA$ and $2.33 \AA$. Structure $(A)$ is the least symmetric structure, with the four base bonds alternating between 2.34 $\AA$ and $2.26 \AA$, and the pyramid bonds are $2.58 \AA$ (twice), $2.68 \AA$ (twice), and $2.32 \AA$ (four times).

All isomers with the tetragonal bipyramidal structure are close in energy and exhibit similar IR spectra. The agreement of the calculated spectra with the experimental one is quite good: the experiments exhibit a well defined peak at $260 \mathrm{~cm}^{-1}$, in agreement with the one at $245 \mathrm{~cm}^{-1}$ in the calculations. The experiment then shows a broad peak around $280 \mathrm{~cm}^{-1}$, which could easily be a double peak. Because of the similarity in the calculated spectra, it is hardly possible to decide which of the isomers $(A)-(C)$ is present, or if a superposition of spectra of several isomers is observed. On the other hand, the spectrum for structure $(D)$ is quite different. (a)
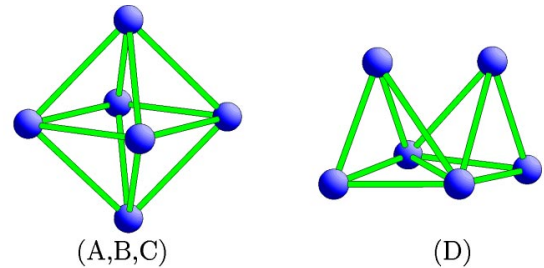

(D)

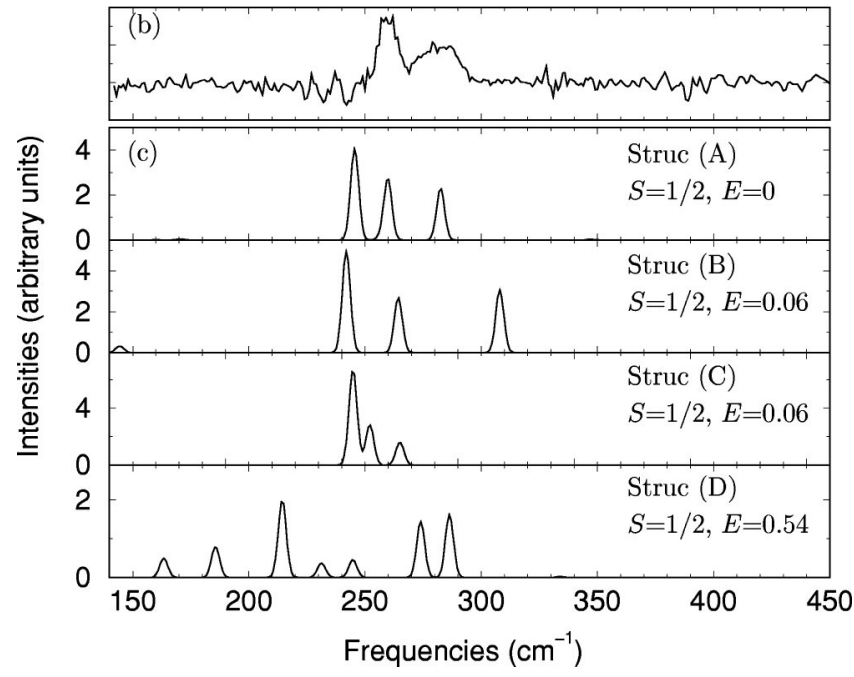

FIG. 8. Results for $\mathrm{V}_{6}^{+}$. (a) Schematic representation of the four most stable structures $(A)-(D)$. Since structures $(A)-(C)$ are very similar (and only differ slightly in bond lengths), only one picture is shown; (b) the spectrum measured experimentally for $\mathrm{V}_{6} \mathrm{Ar}_{2}^{+}$; (c) the spectrum calculated for the structures $(A)-(D)$. The energy differences shown are in $\mathrm{eV}$.

Since structure $(D)$ is also significantly higher in energy, we can rule out this structure as being responsible for the experimental spectrum.

\section{F. Results and discussion for $\mathrm{V}_{7}^{+}$}

The DFT calculations of Grönbeck and Rosén ${ }^{12}$ for $V_{7}$, of $\mathrm{Wu}$ and $\mathrm{Ray}^{13}$ for $\mathrm{V}_{7}^{+}$, and of $\mathrm{Li}$ et al. for $\mathrm{V}_{7}^{+}, \mathrm{V}_{7}^{-}$, and $\mathrm{V}_{7}$, as well as the empirical potential calculations of Sun et al. ${ }^{15}$ all suggest that a (slightly buckled) pentagonal bipyramid is the most stable structure. This is the structure that is shown as structure $(B)$ in Fig. 9. Our calculations indicate that a strongly distorted pentagonal bipyramid as shown in Fig. 9 is in fact the energetically most stable structure. It is $0.06 \mathrm{eV}$ lower than structure $(B)$. Structure $(A)$ could also be described as a tetragonal pyramid with a dimer on the backside of the pyramid (the two atoms most to the right in the figure). This structure $(A)$ is actually similar to the second lowest structure in Refs. 13 and 14.

Comparison of the calculated spectra with the one measured experimentally indicates that structure $(A)$ could indeed be the isomer that is observed in the experiment: the experimental spectrum for $\mathrm{V}_{7} \mathrm{Ar}_{2}^{+}$has a rather broad peak at $315 \mathrm{~cm}^{-1}$, whereas the calculations for structure $(A)$ show one strong peak around $305 \mathrm{~cm}^{-1}$ accompanied by a peak of lower intensity at $315 \mathrm{~cm}^{-1}$. The smaller experimental peak around $268 \mathrm{~cm}^{-1}$ might correspond to the weak feature in the calculations around $275 \mathrm{~cm}^{-1}$. 
(a)

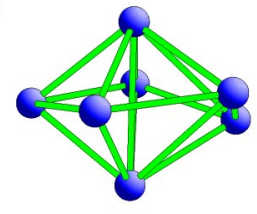

(A)

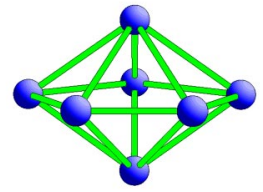

(B)

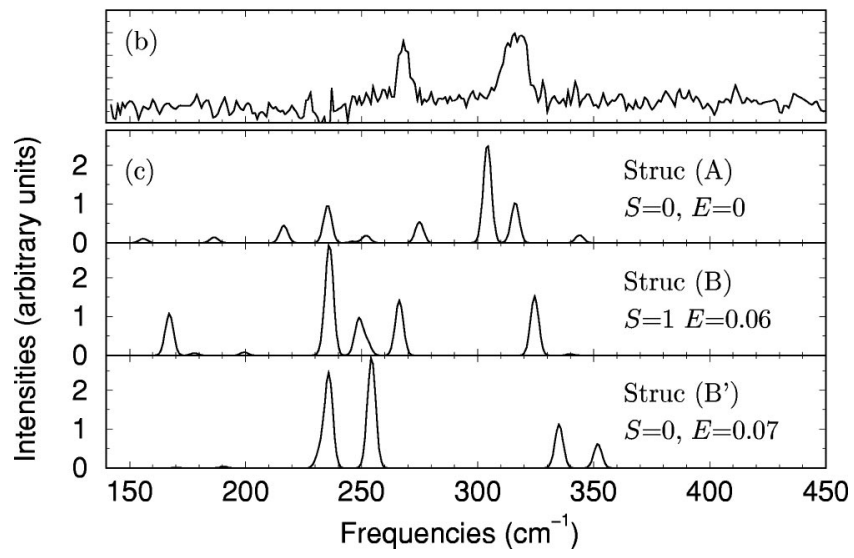

FIG. 9. Results for $\mathrm{V}_{7}^{+}$. (a) Schematic representation of the two most stable structures $(A)$ and $(B)$; (b) the spectrum measured experimentally for $\mathrm{V}_{7} \mathrm{Ar}_{2}^{+}$; (c) the spectrum calculated for the structures $(A),(B)$, and $\left(B^{\prime}\right)$. The energy differences shown are in $\mathrm{eV}$.

\section{G. Results and discussion for $V_{8}^{+}$}

The four most stable structures for $\mathrm{V}_{8}^{+}$are shown in Fig. 10(a). Structure $(A)$ is the same that has been predicted for cationic, ${ }^{13,14}$ anionic, ${ }^{14}$ and neutral ${ }^{12-15} \mathrm{~V}_{8}$ in previous stud-
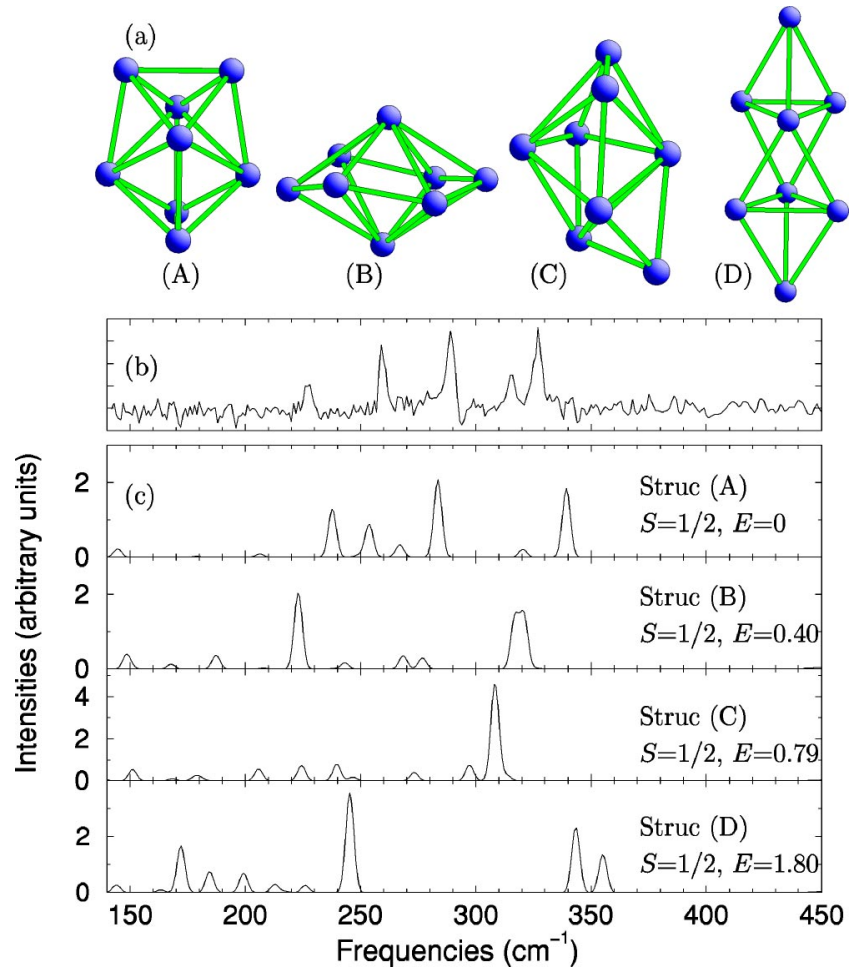

FIG. 10. Results for $\mathrm{V}_{8}^{+}$. (a) Schematic representation of the four most stable structures $(A)-(D)$; (b) the spectrum measured experimentally; (c) the spectrum calculated for the structures $(A)-(D)$. The energy differences shown are in $\mathrm{eV}$.

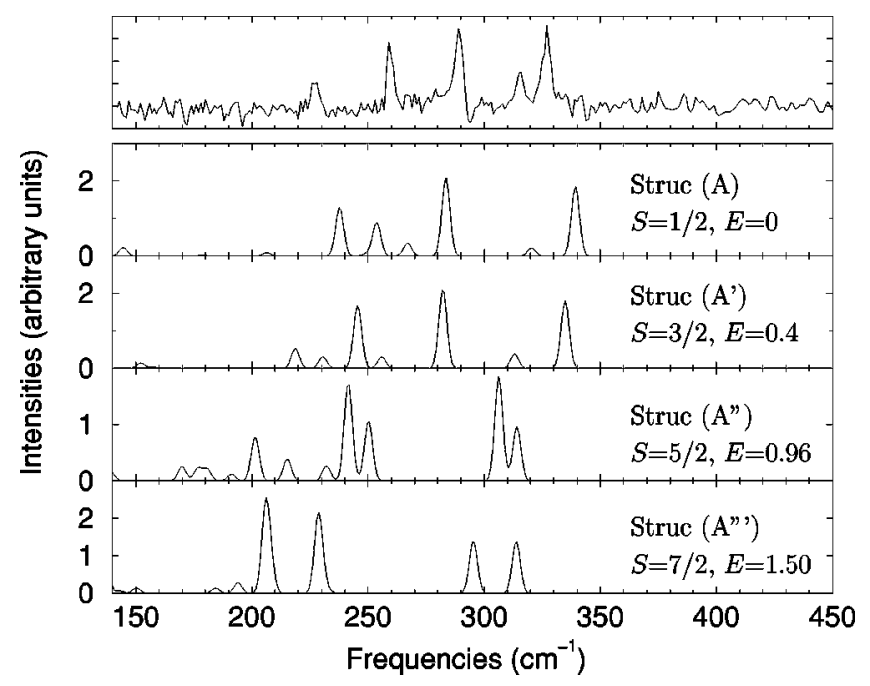

FIG. 11. Calculated spectra obtained with different spins for $\mathrm{V}_{8}^{+}$with structure $(A)$. The energy differences shown are in $\mathrm{eV}$.

ies. It is a combination of a tetragonal bipyramid with two additional atoms capping two neighboring faces. These two atoms then form an additional dimer bond, which is the horizontal (top) bond in Fig. 10(a). The second lowest structure is a buckled hexagonal bipyramid [structure $(B)$ ]. Structure $(C)$ is a variation of structure $(A)$, while structure $(D)$ is a combination of several trigonal pyramids (that has been suggested to be the second lowest structure in Ref. 13). All structures have the lowest energy with spin $S=1 / 2$, but the effect of spin is discussed explicitly below for this cluster for structure $(A)$. The spectrum that has been calculated for structure $(A)$ [cf. Fig. 10(c)] agrees best with the one that is observed experimentally [cf. Fig. 10(b)]. We therefore conclude that this is the structure of the $\mathrm{V}_{8}^{+}$clusters.

In Fig. 11, we compare the spectra calculated for structure (A) with spins of $S=1 / 2, S=3 / 2, S=5 / 2$, and $S=7 / 2$. The spectra for $\mathrm{V}_{8}^{+}$with $S=1 / 2$ and $S=3 / 2$ are very similar and both could be consistent with the experimental spectrum. On the other hand, for larger spins $(S=5 / 2$, and $S=7 / 2)$, the spectrum changes significantly. The energy difference between $S=1 / 2$ and $S=3 / 2$ is $0.42 \mathrm{eV}$, and it increases even more for the higher spin states. On the other hand, as the spin increases, the atomic structure changes only slightly. This minor change in the geometry is manifested as follows: The top dimer bond [the horizontal top bond in Fig. 10(a)] gets shortened and the two corresponding atoms move further away from the faces underneath. Also, as the spin increases, the underlying tetragonal bipyramidal block changes from a very symmetric structure to one that becomes more and more distorted.

From our calculations we conclude that the atomic structure for $\mathrm{V}_{8}^{+}$is the one that is shown as structure $(A)$, and that in fact it has a low spin state. Our calculations suggest $S$ $=1 / 2$.

\section{$H$. Results and discussion for $\mathrm{V}_{9}^{+}$}

We find two structures for $\mathrm{V}_{9}^{+}$that are very similar in energy. They are shown in Fig. 12 as structures $(A)$ and $(B)$. Structure $(A)$ is a cagelike structure. Structure $(B)$ can be 
(a)

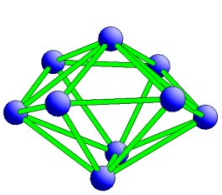

(A)

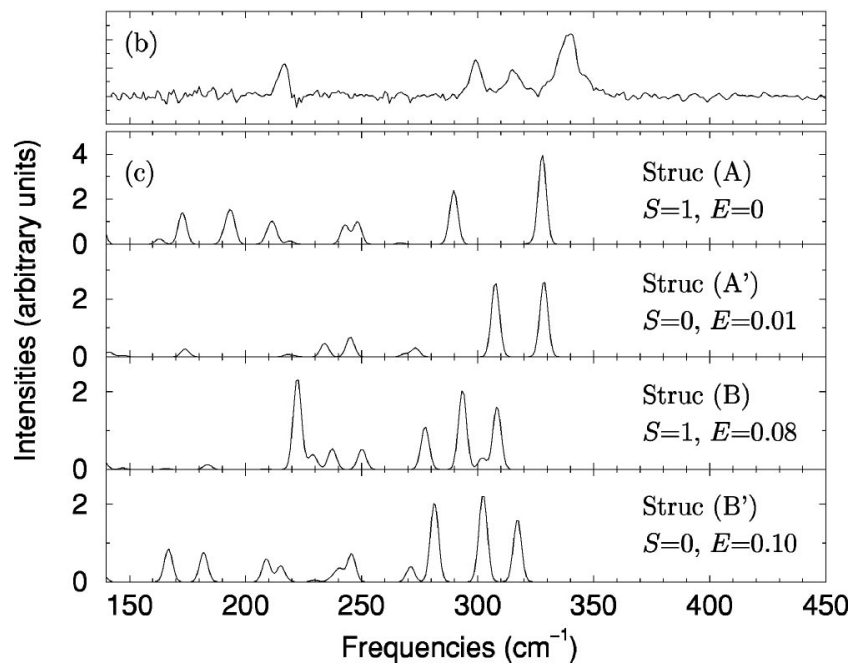

FIG. 12. Results for $\mathrm{V}_{9}^{+}$. (a) Schematic representation of the two most stable structures $(A)-(B)$; (b) the spectrum measured experimentally; (c) the spectrum calculated for the structures $(A),\left(A^{\prime}\right),(B)$, and $\left(B^{\prime}\right)$. The energy differences shown are in $\mathrm{eV}$.

described as a tricapped triganol prism with approximate threefold symmetry. In fact, the geometries of $(A)$ and $(B)$ are not that different: consider the two atoms that are to the far left and right in the picture for structure $(A)$. If one bends these two atoms downwards (and at the same time allows the atoms above to get closer to each other), one arrives at structure $(B)$. We show both structures with both spin states, $S$ $=0$ and $S=1$. The energy differences between all four isomers are very small, and they are essentially degenerate in energy. Thus, we clearly cannot assign the ground state on energetic arguments alone.

We therefore turn our attention to the comparison of the calculated spectra [cf. Fig. 12(c)] with the one obtained experimentally. The experimental spectrum has three well resolved peaks between $300 \mathrm{~cm}^{-1}$ and $350 \mathrm{~cm}^{-1}$, and one additional peak around $215 \mathrm{~cm}^{-1}$. The agreement is best with the spectrum calculated for structure $(B)$, which also exhibits a succession of three strong peaks (between $280 \mathrm{~cm}^{-1}$ and $320 \mathrm{~cm}^{-1}$ ), and one additional strong peak to the red. In contrast, the spectrum of structure $(A)$ does not exhibit these four most prominent peaks. The identification of structure $(B)$ is also in agreement with the results of Ref. 17 for neutral vanadium clusters. We also note that this structure has also been identified as an important building block for silicon clusters. $^{36}$

\section{Results and discussion for $\mathrm{V}_{10}^{+}$}

The most stable structure we found for $\mathrm{V}_{10}^{+}$is very similar to structure $(B)$ for $\mathrm{V}_{9}^{+}$. It can be described as a bicapped anticube, where two stacked tetragons are rotated against each other, and each is topped off by a tetragonal pyramid. It (a)

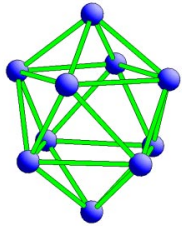

(A)

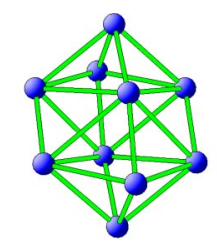

(B)

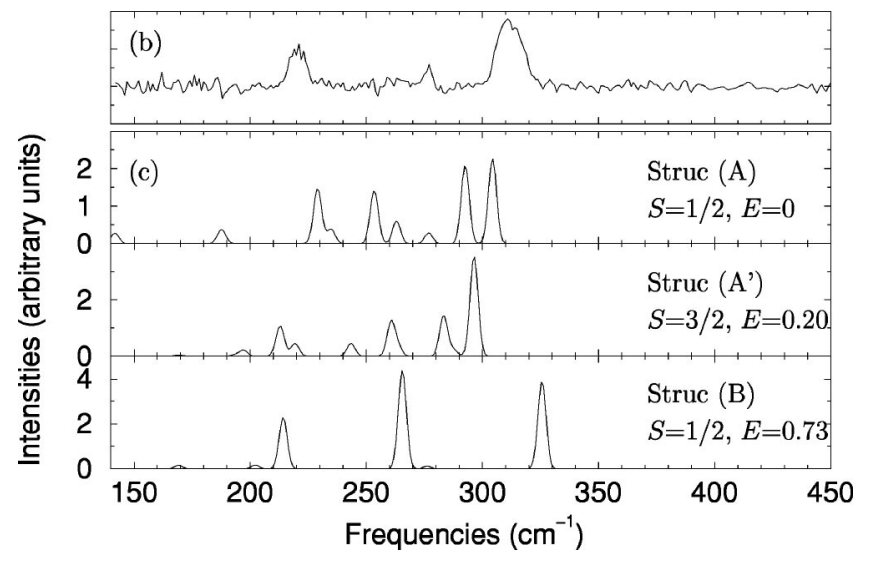

FIG. 13. Results for $\mathrm{V}_{10}^{+}$. (a) Schematic representation of the two most stable structures $(A)-(B) ;(b)$ the spectrum measured experimentally; (c) the spectrum calculated for the structures $(A),\left(A^{\prime}\right)$, and $(B)$. The energy differences shown are in $\mathrm{eV}$.

is shown in Fig. 13(a) as structure $(A)$. It is the same structure that has been found previously ${ }^{17}$ for neutral $\mathrm{V}_{10}$. The energetically preferred spin state is $S=1 / 2$. Its $S=3 / 2$ counterpart $\left(A^{\prime}\right)$ is found to be the second lowest isomer, $0.22 \mathrm{eV}$ higher in energy than isomer $(A)$. An alternative structure $(B)$ is also shown; it can be described as a bicapped cube, where the two stacked tetragons are aligned. It is $\approx 0.72 \mathrm{eV}$ higher in energy.

The proper identification of the structure is difficult. The broad experimental peak between $300 \mathrm{~cm}^{-1}$ and $320 \mathrm{~cm}^{-1}$ might actually be a double peak. Then, the spectrum of structure $(A)$ [and also $\left(A^{\prime}\right)$ ] would agree rather well; for structure (A) we find two peaks just above and below $300 \mathrm{~cm}^{-1}$. The experiment then shows another prominent peak at $221 \mathrm{~cm}^{-1}$, with an additional weaker peak at $277 \mathrm{~cm}^{-1}$. These peaks can also be matched up with the ones obtained for structure $(A)$. The spectrum for structure $(B)$ is rather different, and shows only three distinct peaks. The energy of isomer $(B)$ is significantly higher (more than the uncertainty of the calculations), and we therefore suggest that $\mathrm{V}_{10}^{+}$prefers to have the structure of isomer $(A)$.

\section{$J$. Results and discussion for $\mathrm{V}_{11}^{+}$}

The energetically preferred structures for $\mathrm{V}_{11}^{+}, \mathrm{V}_{12}^{+}$, and $\mathrm{V}_{13}^{+}$are all very similar: they are all related to the structure of an icosahedron, and can be described as consisting of two pentagons, that are stacked above each other, and are rotated against each other. Then, one, two, or three more atoms are added to build pentagonal pyramids, and/or are located in the center. For example, the structure with the lowest energy for $\mathrm{V}_{11}^{+}$is shown in Fig. 14(a) as structure $(A)$. It consists of two (distorted) pentagons with a pentagonal pyramid on top. 
(a)

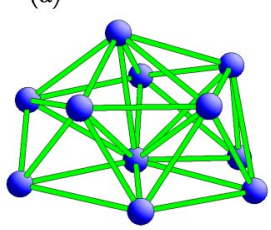

(A)

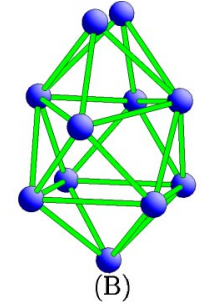

(B)
(C)
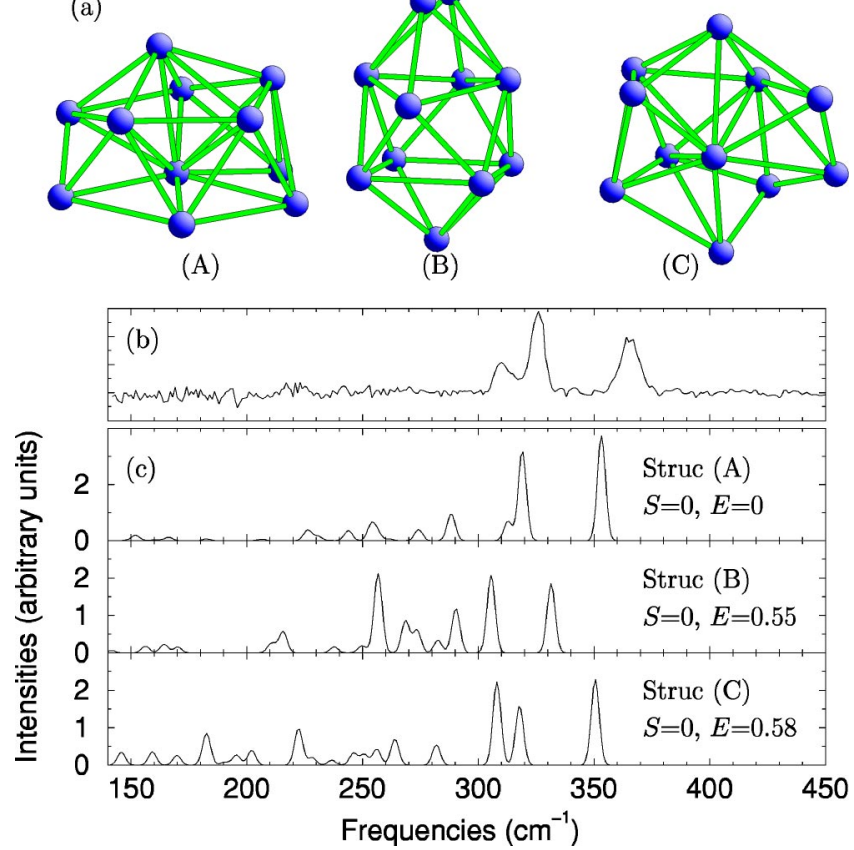

FIG. 14. Results for $\mathrm{V}_{11}^{+}$. (a) Schematic representation of the three most stable structures $(A)-(C)$; (b) the spectrum measured experimentally; (c) the spectrum calculated for the structures $(A)-(C)$. The energy differences shown are in $\mathrm{eV}$.

The calculated spectrum for structure $(A)$ as shown in Fig. 14(c) agrees very well with the one that has been measured in the experiment [cf. Fig. 14(b)]. The alternative structure with the second lowest energy is structure $(B)$. It is based on two tetragons [similar to structure $(A)$ for $\mathrm{V}_{10}^{+}$and structure $(B)$ for $\left.\mathrm{V}_{9}^{+}\right]$. It is significantly higher in energy, and exhibits a spectrum that is quite different. We therefore conclude that the structure observed experimentally might be the one that is shown as structure $(A)$. We also show an additional, cagelike structure $(C)$ that is slightly higher in energy. All structures shown have the lowest energy for a spin of $S=0$.

\section{K. Results and discussion for $\mathrm{V}_{12}^{+}$}

The experimental spectrum for $\mathrm{V}_{12}^{+}$reveals very distinct peaks at $180 \mathrm{~cm}^{-1}, 285 \mathrm{~cm}^{-1}$, and $307 \mathrm{~cm}^{-1}$, and a rather broad peak (that could easily be a double peak) around $354 \mathrm{~cm}^{-1}$. Structure $(A)$ is the lowest energy structure: it is a rather symmetric, icosahedral structure. As mentioned above, it is similar to structure $(A)$ for $\mathrm{V}_{11}^{+}$, except that it has two pentagonal pyramids. The calculations for this isomer give two peaks that match very well the experimental peaks at $285 \mathrm{~cm}^{-1}$ and $307 \mathrm{~cm}^{-1}$ [cf. Fig. 15(c)]. However, no other peaks are obtained. Note that the double-peak structure is due to a slight structural distortion from the perfect icosahedron which has only one IR active mode.

Structure $(B)$ with $S=1 / 2$ is only slightly higher in energy $(0.12 \mathrm{eV})$. It also consists of two (rotated) pentagons, but only one pentagonal pyramid, and one additional atom in the center. This structure has in fact been predicted to be the energetically preferred structure for neutral $\mathrm{V}_{12}$ in a previous study. ${ }^{17}$ It has two additional peaks between $350 \mathrm{~cm}^{-1}$ and
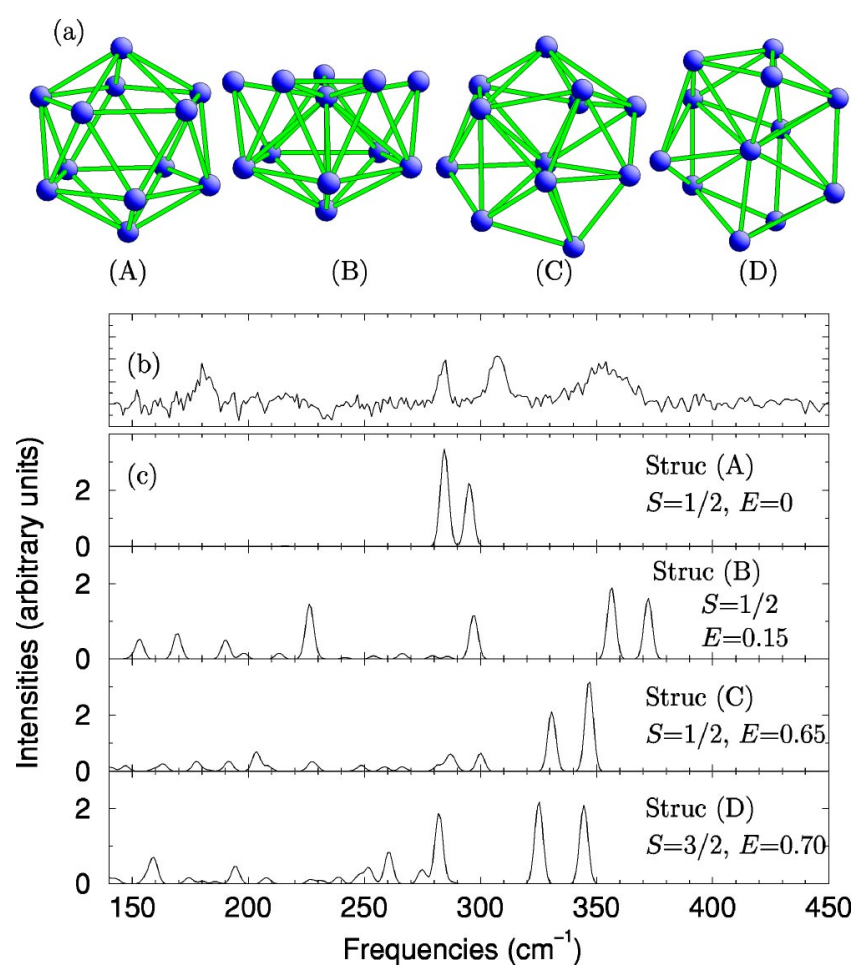

FIG. 15. Results for $\mathrm{V}_{12}^{+}$. (a) Schematic representation of the four most stable structures $(A)-(D)$; (b) the spectrum measured experimentally; (c) the spectrum calculated for the structures $(A)-(D)$. The energy differences shown are in $\mathrm{eV}$.

$380 \mathrm{~cm}^{-1}$, matching the broad (double) peak that is observed experimentally. In addition, this structure exhibits a peak around $220 \mathrm{~cm}^{-1}$, which could correspond to the experimental peak in the low frequency range. Both structures $(A)$ and $(B)$ are related to the highly symmetric icosahedral structure (A) for $\mathrm{V}_{13}^{+}$(cf. Sec. IV L): Removing the center atom from structure $(A)$ of $\mathrm{V}_{13}^{+}$leads to structure $(A)$ for $\mathrm{V}_{12}^{+}$, while removing one of the top (or bottom) outside atoms leads to structure $(B)$. We also show structures $(C)$ with $S=1 / 2$ and $(D)$ with $S=3 / 2$ that are almost degenerate in energy, and are about $0.6-0.7 \mathrm{eV}$ higher than $(A)$. Both also exhibit two significant peaks between $320 \mathrm{~cm}^{-1}$ and $360 \mathrm{~cm}^{-1}$.

The experimental spectrum cannot be reproduced by a calculated spectrum of any single isomer. We therefore suggest that the spectrum observed experimentally is due to a superposition of the spectra of the different isomers shown in Fig. 15(a). Based on the calculated energy differences our calculations suggest that it might be a superposition of the spectra of the structures $(A)$ and $(B)$. We note that the presence of different isomers in this size range is well established, for example, for neutral and cationic niobium clusters. ${ }^{37}$

\section{Results and discussion for $V_{13}^{+}$}

Structure $(A)$ as shown in Fig. 16(a) is the energetically preferred structure for $\mathrm{V}_{13}^{+}$. It is an icosahedral structure with one atom at the center. We also show two additional structures in Fig. 16(a), and the corresponding spectra in Fig. 16(c). Structure $(B)$ is a rather asymmetric structure. Its building blocks are a centered hexagon in the middle, with 


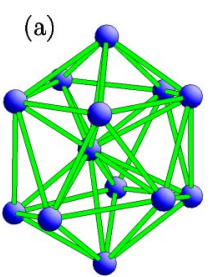

(A)

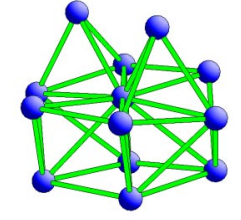

(B)

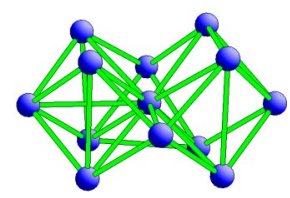

(C)

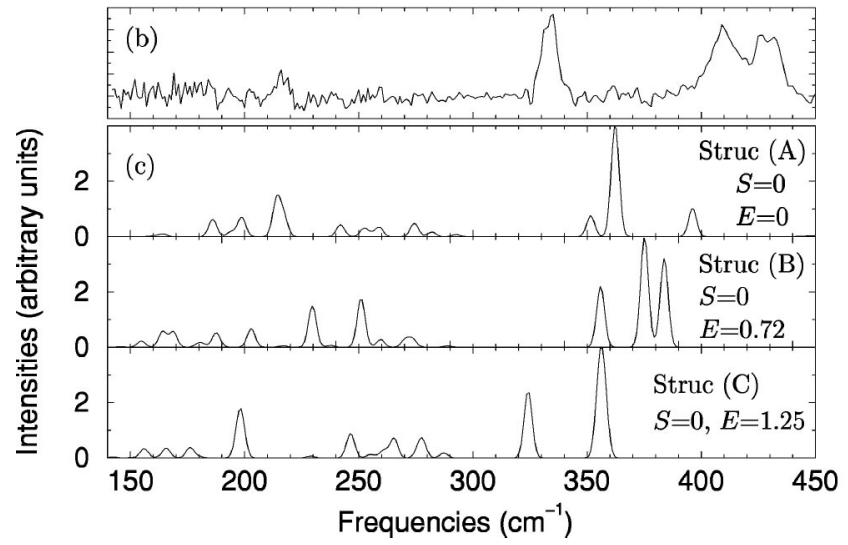

FIG. 16. Results for $\mathrm{V}_{13}^{+}$. (a) Schematic representation of the three most stable structures $(A)-(C)$; (b) the spectrum measured experimentally; (c) the spectrum calculated for the structures $(A)-(C)$. The energy differences shown are in $\mathrm{eV}$.

two atoms (forming distorted pyramids) above, and four atoms below. It is $0.71 \mathrm{eV}$ higher in energy than structure $(A)$. Shown is also structure $(C)$, which is even higher in energy, and has a different spectrum. Nevertheless, this is an interesting structure worth discussing. It is related to the bulklike structures $(B)$ for $\mathrm{V}_{14}^{+}$and $(A)$ for $\mathrm{V}_{15}^{+}$.

Experimentally, the spectrum of $\mathrm{V}_{13}^{+}$is interesting: It is the only spectrum for cluster sizes between 3 and 15 atoms where we see peaks at wave numbers higher than $400 \mathrm{~cm}^{-1}$ [Fig. 16(b)]. Such a high vibrational frequency can be a signature of a mode that involves a strongly bound atom. Our calculations for the centered structure $(A)$ do predict a peak around $400 \mathrm{~cm}^{-1}$, which is the highest frequency IR active mode that we calculate for any structure. Inspection of the calculated eigenvectors indicates that this mode is associated with the center atom. In a perfect icosahedron, the lengths of the bonds of the central atom are expected to be about $5 \%$ shorter than the bonds among the surrounding atoms, which leads to an increase of the stiffness. We actually find that this center atom is not symmetrically in the middle of the structure; in fact, several of its bonds are much shorter than its average bond length (by $\approx 10 \%$ ). This increased stiffness could be the reason for the high frequency mode. In addition, we find a strong peak just above $350 \mathrm{~cm}^{-1}$, that can be matched with the experimental peak just below $350 \mathrm{~cm}^{-1}$, and one additional weaker peak around $220 \mathrm{~cm}^{-1}$, that is also seen experimentally. A double peak around $380 \mathrm{~cm}^{-1}$ is found for isomer $(B)$, but this is quite strongly shifted in comparison to the experimental double peak.

The experimental peaks around $420 \mathrm{~cm}^{-1}$ are not reproduced by any of the calculated isomers. The most likely match is structure $(A)$, but as indicated, we only find one peak at large frequencies above $400 \mathrm{~cm}^{-1}$, and even that

(a)
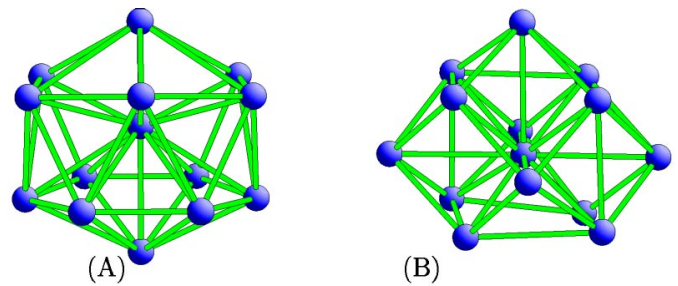

(B)

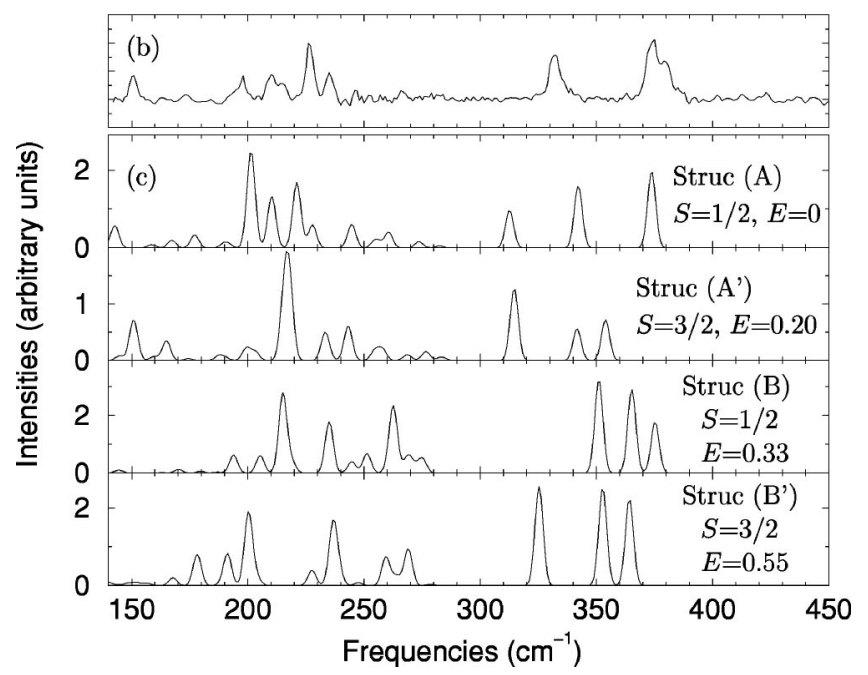

FIG. 17. Results for $\mathrm{V}_{14}^{+}$. (a) Schematic representation of the two most stable structures $(A)-(B)$; (b) the spectrum measured experimentally; (c) the spectrum calculated for the structures $(A)-(B)$. The energy differences shown are in eV.

single peak is calculated with far less intensity. We have also verified that structure $(A)$ with higher spin states does not have a lower ground state energy, as it has been suggested, for example, for lithium clusters. ${ }^{38,39}$

\section{Results and discussion for $\mathrm{V}_{14}^{+}$}

The experimental spectrum of $\mathrm{V}_{14}^{+}$shows much more structure than the spectra for $\mathrm{V}_{13}^{+}$or $\mathrm{V}_{15}^{+}$. Just in the range between 140 and $250 \mathrm{~cm}^{-1}$ at least seven intense peaks can be identified. This high number of IR active modes suggests a lower symmetry for $\mathrm{V}_{14}^{+}$in comparison to the neighboring sizes (or the presence of several isomers). As discussed before (cf. Sec. IV L), the structure for $\mathrm{V}_{13}^{+}$might be the (slightly distorted) icosahedron. As will be discussed below, we suggest that $\mathrm{V}_{15}^{+}$has the structure of a bulklike capped cube. These are both highly symmetric structures. The structure of $\mathrm{V}_{14}^{+}$might then be related to either of these structure by simply adding or removing a $\mathrm{V}$ atom, respectively. We also find that similar to $\mathrm{V}_{13}^{+}$, a rather high frequency mode appears.

In our calculations for $\mathrm{V}_{14}^{+}$, two structures emerge, that are shown in Fig. 17(a). Structure $(A)$ is a cagelike structure that can be described as follows: it is a stack of a hexagon and a pentagon, with two additional atoms above and below, and one more in the center. Structure $(B)$ is very bulklike: the basic elements of this structure are a body-centered cube $(8$ +1 atoms), plus five additional atoms that form tetragonal pyramids on five of the six faces of the cube. Both structures are related to the highly symmetric structures $(A)$ and $(B)$ we find for $\mathrm{V}_{15}^{+}$. The difference is that for structure $(A)$, one 

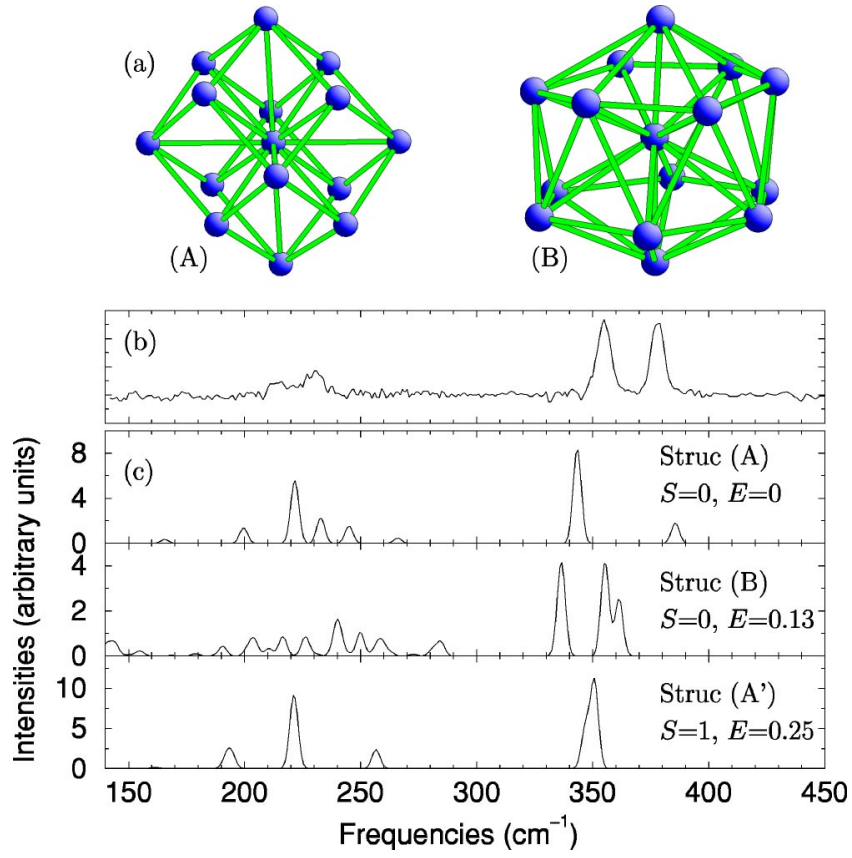

FIG. 18. Results for $\mathrm{V}_{15}^{+}$. (a) Schematic representation of the two most stable structures $(A)-(B)$; (b) the spectrum measured experimentally; (c) the spectrum calculated for the structures $(A),(B)$, and $\left(A^{\prime}\right)$. The energy differences shown are in $\mathrm{eV}$.

hexagon is replaced by a pentagon, and that for structure $(B)$ there is one outside atom less [compared to structure $(A)$ for $\mathrm{V}_{15}^{+}$. Both structures include a central atom.

All the spectra calculated for structures $(A)$ and $(B)$ with spins $S=1 / 2$ and $3 / 2$ exhibit a number of peaks between $170 \mathrm{~cm}^{-1}$ and $270 \mathrm{~cm}^{-1}$, which can be matched to the large number of peaks in the experimental spectrum between $140 \mathrm{~cm}^{-1}$ and $250 \mathrm{~cm}^{-1}$. Therefore, we will focus the comparison on the features at higher frequencies: The experimental spectrum has a peak at $330 \mathrm{~cm}^{-1}$, and a broader peak between $370 \mathrm{~cm}^{-1}$ and $380 \mathrm{~cm}^{-1}$, which most likely is a double peak. Structures $(A)$ and $(B)$ for both spin values exhibit 3 peaks above $300 \mathrm{~cm}^{-1}$. We verified that similar to $\mathrm{V}_{13}^{+}$, the highest frequency mode corresponds to a vibration of the central atom (for both structures). The spectrum of structure $(A)$ also agrees qualitatively in the low frequency region. Here, relative intensities differ between experiment and theory. Nonetheless, based on the overall good agreement, structure $(A)$ is the isomer that is most likely observed in the experiment.

\section{N. Results and discussion for $\mathrm{V}_{15}^{+}$}

In contrast to $\mathrm{V}_{14}^{+}$, the experimental spectrum of $\mathrm{V}_{15}^{+}$ shows very few peaks. This is an indication for a highly symmetric structure. Our calculations suggest two structures that are shown in Fig. 18(a) and that are indeed very symmetric. Structure $(A)$ is a bulklike structure, consisting of a body-centered cube $(8+1$ atoms $)$ plus 6 more atoms forming tetragonal pyramid above each face. Structure $(B)$ consists of two hexagons that are rotated with a center atom and two more atoms above and below (forming hexagonal pyramids). The energy difference between structures $(A)$ and $(B)$ for spin $S=0$ is rather small, only about $0.13 \mathrm{eV}$.
The spectra for both isomers have a peak just below $350 \mathrm{~cm}^{-1}$, that matches an experimental peak at $355 \mathrm{~cm}^{-1}$. Structure $(A)$ then also exhibits another (weaker) peak around $385 \mathrm{~cm}^{-1}$, while structure $(B)$ has two more peaks to the high-frequency side between $350 \mathrm{~cm}^{-1}$ and $370 \mathrm{~cm}^{-1}$. The experimental spectrum has a second strong peak at $379 \mathrm{~cm}^{-1}$. This is a rather narrow, well-defined peak, and does not appear to be a double peak. The agreement with the spectrum of the bulklike structure $(A)$ is slightly better, but we cannot say this with certainty. Both, structures $(A)$ and (B) exhibit a few peaks with low intensities below $300 \mathrm{~cm}^{-1}$ that match the experimental peaks at $213 \mathrm{~cm}^{-1}$ and $231 \mathrm{~cm}^{-1}$.

\section{CONCLUSIONS}

We have shown that the atomic structure of small metal clusters can be identified by a comparison of experimental far infrared spectra with vibrational spectra calculated with DFT. We have presented systematic results for cationic vanadium clusters in the size range from 3 to 15 atoms. For most clusters, we were able to identify the atomic structure of the cluster. In particular, we have shown that knowledge of the ground state energy alone is not enough. In some cases (such as $\mathrm{V}_{5}^{+}$) it appears that the structure that is observed in the experiment is actually not the one that has the lowest energy in the calculations. In several other cases, the energy difference between different structures is very small, and in fact negligible within the accuracy of a DFT calculation, so that an unambiguous identification of the structure based on energetic arguments is not possible. However, comparison of the calculated vibrational spectra with the experimentally measured IR spectra does allow for the identification of the atomic structure. In some cases (such as $\mathrm{V}_{12}^{+}$), it appears that the observed experimental spectrum is not the result of just one isomer, and that in fact several different atomic structures might be present.

For clusters with 13 and more vanadium atoms, a significant change occurs in the high wave number range of the spectra, that can be correlated with a structural transition. Clusters up to size 12 only consist of surface atoms. Starting at size 13, the structure of the cluster includes a central atom. This central atom is bound stronger than the other atoms, leading to a higher vibrational frequency. As the cluster size increases, the structures are less constrained. Thus, they weaken their bonds to the central atom, which affects the respective bond length and leads to a slight lowering of the frequency of the highest frequency mode observed. Close inspection of the eigenvectors reveals that for clusters of sizes 13-15, the highest frequency mode does indeed involve a vibration of the central atom.

For a few cluster sizes, we have not been able to unambiguously identify the atomic structure. This problem becomes more serious as the cluster size increases. For larger clusters, mixtures of isomers can be present in the experiment and, on the other hand, theory can miss relevant structures. Ultimately, more sophisticated computational schemes are required to test a large number of different structures, with different distortions and different spin states. Neverthe- 
TABLE II. Convergence of the total energy of a vanadium dimer and its vibrational frequency as a function of the basis set. All basis sets include also the $1 s, 2 s, 2 p, 3 s$, and $3 p$ orbitals. The additional basis for sets 1-8 are given in parentheses. The superscript $* *$ denotes the fact that these are orbitals that are typically used for titanium. For all tests in this table, we used a cutoff radius of 12.0 bohrs, and a mesh parameter of 1.6.

\begin{tabular}{clcr}
\hline \hline $\begin{array}{c}\text { Convergence } \\
\text { test label }\end{array}$ & $\begin{array}{l}\text { Number (character) } \\
\text { of basis functions }\end{array}$ & $\begin{array}{c}\text { Energy } \\
(\mathrm{eV})\end{array}$ & $\begin{array}{c}\text { Frequency } \\
\left(\mathrm{cm}^{-1}\right)\end{array}$ \\
\hline 1 & $18(4 p)$ & -51816.262 & 673.2 \\
2 & $21(4 s)$ & -51816.230 & 570.1 \\
3 & $21(3 d)$ & -51816.578 & 599.0 \\
4 & $24(4 s 4 p)$ & -51816.727 & 594.7 \\
5 & $24(4 s 4 p 3 d)$ & -51817.000 & 617.6 \\
6 & $25(5 s)$ & -51817.004 & 621.7 \\
7 & $28(5 p)$ & -51817.027 & 618.2 \\
8 & $33\left(4 s^{* *} 4 p^{* *} 3 d^{* *}\right)$ & -51817.184 & 632.5 \\
\hline \hline
\end{tabular}

less, the work described in this paper provides one step further in the computational prediction and identification of the atomic structure of small metal clusters. We believe that it is straightforward to extend this approach to other types of nanoparticles, and that much new insight on the size dependent evolution of cluster properties can be gained.

\section{ACKNOWLEDGMENTS}

This work is a part of the research program of the "Stichting voor Fundamenteel Onderzoek der Materie" (FOM), which was supported financially by the "Nederlandse organisatie voor Wetenschappelijk Onderzoek" (NWO). Financial support from the EU IHP Research Training Network (Delayed Ionisation and Competing Cooling Mechanisms in Atomic Clusters) is gratefully acknowledged. The authors also thank Accelrys for providing the $\mathrm{DMOL}^{3}$ software to the California NanoSystems Institute.

\section{APPENDIX: CONVERGENCE TESTS FOR THE DMOL ${ }^{3}$ CODE}

$\mathrm{DMOL}^{3}$ is an all-electron DFT code that uses atomic orbitals as a basis set. ${ }^{40}$ For all calculations presented, a basis set consisting of 24 basis functions was used. Convergence tests of the total energy and the vibrational frequency of a (neutral) vanadium dimer with respect to the number and character of the basis functions are shown in Table II. Convergence

TABLE III. Convergence of the total energy of a vanadium dimer and its vibrational frequency as a function of the cutoff radius. For all tests in this table, we used 24 basis functions (convergence test 5 ) and a mesh parameter of 1.6 .

\begin{tabular}{ccc}
\hline \hline $\begin{array}{c}r_{\text {cut }} \\
(\text { Bohr })\end{array}$ & $\begin{array}{c}\text { Energy } \\
(\mathrm{eV})\end{array}$ & $\begin{array}{c}\text { Frequency } \\
\left(\mathrm{cm}^{-1}\right)\end{array}$ \\
\hline 8.0 & -51816.477 & 632.8 \\
9.0 & -51816.785 & 623.2 \\
10.0 & -51816.918 & 621.5 \\
11.0 & -51816.973 & 620.0 \\
12.0 & -51817.000 & 617.6 \\
13.0 & -51817.008 & 619.2 \\
14.0 & -51817.008 & 621.1 \\
\hline \hline
\end{tabular}

TABLE IV. Convergence of the total energy of a vanadium dimer and its vibrational frequency as a function of the computational mesh. For all tests in this table, we used 24 basis functions (convergence test 5) and a cutoff radius of 12.0 bohrs.

\begin{tabular}{ccc}
\hline \hline Mesh & $\begin{array}{c}\text { Energy } \\
(\mathrm{eV})\end{array}$ & $\begin{array}{c}\text { Frequency } \\
\left(\mathrm{cm}^{-1}\right)\end{array}$ \\
\hline 1.0 & -51816.988 & 623.0 \\
1.2 & -51817.004 & 618.4 \\
1.4 & -51816.996 & 618.8 \\
1.6 & -51817.000 & 617.6 \\
1.8 & -51817.000 & 620.1 \\
2.0 & -51816.996 & 620.0 \\
\hline
\end{tabular}

test 5 includes the $4 s, 4 p$, and $3 d$ orbitals, and is referred to as basis set all in the code. Including a smaller number of orbitals (convergence tests 1-4) does not appear to be sufficient. On the other hand, the change in total energy and frequency is rather small when more orbitals are added to the basis set (convergence tests 6 and 7). We have also tried adding completely different orbitals: Adding the $4 \mathrm{~s}, 4 \mathrm{p}$, and $3 \mathrm{~d}$ orbitals of Ti gives 33 basis functions (test 8 ). The total energy only changes by about $20 \mathrm{meV}$, and the frequency changes by no more than $2 \%$. Note that the energy given is the total energy of the dimer. The effect of the basis set on energy differences will be substantially smaller, so that a basis set consisting of 24 basis functions per atom (test 5) is sufficient to describe vanadium.

The basis functions are truncated at a real space cut-off radius of 12 bohrs. Convergence with respect to this cutoff radius is shown in Table III To numerically evaluate the required matrix elements a dense grid of integration points in real space has been used. ${ }^{40}$ The grid points for the numerical integration are arranged on atom-centered spheres (with logarithmic spacing) according to the scheme suggested by Lebedev. $^{41}$ The mesh parameter determines the number of spheres, and convergence with respect to this parameter is shown in Table IV. A mesh parameter of 1.6 has been used for all calculations in this paper, which corresponds to 65 spheres for each vanadium atom. We have also tested that a sufficiently large number of integration points on, each sphere are used, and we have used 11912 integration points per vanadium atom.

${ }^{1}$ I. M. L. Billas, J. A. Becker, A. Chatelain, and W. A. de Heer, Phys. Rev. Lett. 71, 4067 (1993).

${ }^{2}$ F. Liu, S. N. Khanna, and P. Jena, Phys. Rev. B 43, 8179 (1991).

${ }^{3}$ K. Lee and J. Callaway, Phys. Rev. B 48, 15358 (1993).

${ }^{4}$ P. Alvarado, J. Dorantes-Dávila, and H. Dreyssé, Phys. Rev. B 50, 1039 (1994).

${ }^{5}$ H. Wu, S. R. Desai, and L.-S. Wang, Phys. Rev. Lett. 77, 2436 (1996).

${ }^{6}$ M. Iseda, T. Nishio, S. Y. Han, H. Yoshida, A. Terasaki, and T. Kondow, J. Chem. Phys. 106, 2182 (1997).

${ }^{7}$ M. Valden, X. Lai, and D. W. Goodman, Science 281, 1647 (1998).

${ }^{8}$ A. T. Bell, Science 299, 1688 (2003).

${ }^{9}$ P. Weis, T. Bierweiler, S. Gilb, and M. M. Kappes, Chem. Phys. Lett. 355, 355 (2002); S. Gilb, P. Weis, F. Furche, R. Ahlrichs, and M. M. Kappes, J. Chem. Phys. 116, 4094 (2002); F. Furche, R. Ahlrichs, P. Weis, C. Jacob, S. Gilb, T. Bierweiler, and M. M. Kappes, ibid. 117, 6982 (2002).

${ }^{10}$ K. A. Bosnick, T. L. Haslett, S. Fedrigo, M. Moskovits, W.-T. Chan, and R. Fournier, J. Chem. Phys. 111, 8867 (1999). 
${ }^{11}$ J. R. Lombardi and B. Davis, Chem. Rev. (Washington, D.C.) 102, 2431 (2002); B. Zhao, H. Lu, J. Jules, and J. R. Lombardi, Chem. Phys. Lett 362, 90 (2002).

${ }^{12}$ H. Grönbeck and A. Rosén, J. Chem. Phys. 107, 10620 (1997).

${ }^{13}$ X. Wu and A. K. Ray, J. Chem. Phys. 110, 2437 (1998).

${ }^{14} \mathrm{~S}$. Li, M. M. G. Alemany, and J. R. Chelikowsky, J. Chem. Phys. 121 5895 (2004).

${ }^{15}$ H. Sun, Y.-H. Luo, J. Zhao, and G. Wang, Phys. Status Solidi B 215, 1127 (1999).

${ }^{16}$ J. Zhao, X. Chen, Q. Sun, F. Liu, G. Wang, and K. D. Lain, Physica B 215, 377 (1995).

${ }^{17}$ A. Tanedo, T. Shimizu, and Y. Kawazoe, J. Phys.: Condens. Matter 13, L305 (2001).

${ }^{18}$ A. Fielicke, A. Kirilyuk, C. Ratsch, J. Behler, M. Scheffler, G. von Helden, and G. Meijer, Phys. Rev. Lett. 93, 023401 (2004).

${ }^{19}$ G. von Helden, D. van Heijnsbergen, and G. Meijer, J. Phys. Chem. 107, 1671 (2003).

${ }^{20}$ A. Fielicke, G. von Helden, and G. Meijer, Eur. Phys. J. D (in press).

${ }^{21}$ A. Schweizer, J. M. Weber, S. Gilb, H. Schneider, D. Schooss, and M. M. Kappes, J. Chem. Phys. 119, 3699 (2003).

${ }^{22}$ L. W. Bruch, M. W. Cole, and E. Zaremba, Physical Adsorption: Forces and Phenomena (Clarendon, Oxford, 1997), p. 229.

${ }^{23}$ J. Oomens, A. G. G. Tielens, B. Sartakov, G. von Helden, and G. Meijer, Astrophys. J. 591, 968 (2003).

${ }^{24}$ B. Delley, J. Chem. Phys. 92, 508 (1990)

${ }^{25}$ J. P. Perdew, K. Burke, and M. Ernzerhof, Phys. Rev. Lett. 77, 3865 (1996).

${ }^{26}$ B. Hammer, L. B. Hansen, and J. K. Nørskov, Phys. Rev. B 59, 7413 (1999).
${ }^{27}$ J. P. Perdew and Y. Wang, Phys. Rev. B 45, 13244 (1992)

${ }^{28}$ P. Calaminici, A. M. Köster, J. Tucker Carrington, P. N. Roy, N. Russo, and D. R. Salahub, Chem. Phys. 114, 4036 (2001).

${ }^{29}$ C. Cossé, M. Fouassier, T. Mejean, M. Tranquille, D. P. DiLella, and M. Moskovits, J. Chem. Phys. 73, 6076 (1980).

${ }^{30}$ P. R. R. Langridge-Smith, M. D. Morse, G. P. Hansen, R. E. Smalley, and A. J. Merer, J. Chem. Phys. 80, 593 (1984).

${ }^{31}$ D. S. Yang, A. M. James, D. M. Rayner, and P. A. Hackett, J. Chem. Phys. 106, 3129 (1995)

${ }^{32}$ Footnote 35 in R. Moro, X. Xu, S. Yin, and W. A. de Heer, Science 300, 1265 (2003)

${ }^{33}$ D. S. Yang, A. M. James, D. M. Rayner, and P. A. Hackett, Chem. Phys. Lett. 231, 177 (1994)

${ }^{34}$ B. Simard, S. A. Mitchell, D. M. Rayner, in Metal-Ligand Interactions in Chemistry, Physics and Biology, NATO Science Series C; Mathematical and Physical Sciences, edited by N. Russo and D. R. Salahub (Kluwer Academic, Dordrecht, 2000), Vol. 546, pp. 239-294.

${ }^{35} \mathrm{~S}$. Minemoto, A. Terasaki, and T. Kondow, J. Electron Spectrosc. Relat. Phenom. 106, 171 (2000)

${ }^{36}$ S. Nigam, C. Majumder, and S. K. Kulshreshtha, J. Chem. Phys. 121, 7756 (2004).

${ }^{37}$ A. Bérces, P. A. Hackett, L. Lian, S. A. Mitchell, and D. M. Rayner, J. Chem. Phys. 108, 5476 (1998), and references therein.

${ }^{38}$ G. Gardet, F. Rogemond, and H. Chermette, J. Chem. Phys. 105, 9933 (1996).

${ }^{39}$ R. Fournier, J. B. Y. Cheng, and A. Wong, J. Chem. Phys. 119, 9444 (2003).

${ }^{40}$ B. Delley, J. Comput. Chem. 17, 1152 (1996).

${ }^{41}$ V. I. Lebedev, Zh. Vychisl. Mat. Mat. Fiz. 15, 48 (1975); 16, 293 (1976). 